\title{
Recent Advances in Dimensionality Reduction Modeling and Multistability Reconstitution of Memristive Circuit
}

\author{
Yunzhen Zhang $\mathbb{D}^{1}{ }^{1}$ Yuan Ping $\mathbb{D}^{1},{ }^{1}$ Zhili Zhang $\mathbb{D}^{1},{ }^{1}$ and Guangzhe Zhao $\mathbb{D}^{2}$ \\ ${ }^{1}$ School of Information Engineering, Xuchang University, Xuchang 461000, China \\ ${ }^{2}$ Academic Administration, Xuchang University, Xuchang 461000, China \\ Correspondence should be addressed to Yunzhen Zhang; yunzhenzhang@xcu.edu.cn
}

Received 20 May 2021; Accepted 23 June 2021; Published 9 July 2021

Academic Editor: Cristiana J. Silva

Copyright (c) 2021 Yunzhen Zhang et al. This is an open access article distributed under the Creative Commons Attribution License, which permits unrestricted use, distribution, and reproduction in any medium, provided the original work is properly cited.

\begin{abstract}
Due to the introduction of memristors, the memristor-based nonlinear oscillator circuits readily present the state initial-dependent multistability (or extreme multistability), i.e., coexisting multiple attractors (or coexisting infinitely many attractors). The dimensionality reduction modeling for a memristive circuit is carried out to realize accurate prediction, quantitative analysis, and physical control of its multistability, which has become one of the hottest research topics in the field of information science. Based on these considerations, this paper briefly reviews the specific multistability phenomenon generating from the memristive circuit in the voltage-current domain and expounds the multistability control strategy. Then, this paper introduces the accurate flux-charge constitutive relation of memristors. Afterwards, the dimensionality reduction modeling method of the memristive circuits, i.e., the incremental flux-charge analysis method, is emphatically introduced, whose core idea is to implement the explicit expressions of the initial conditions in the flux-charge model and to discuss the feasibility and effectiveness of the multistability reconstitution of the memristive circuits using their flux-charge models. Furthermore, the incremental integral transformation method for modeling of the memristive system is reviewed by following the idea of the incremental flux-charge analysis method. The theory and application promotion of the dimensionality reduction modeling and multistability reconstitution are proceeded, and the application prospect is prospected by taking the synchronization application of the memristor-coupled system as an example.
\end{abstract}

\section{Introduction}

The intrinsic memory property [1] of the memristor makes the memristor-based nonlinear circuits and systems easily exhibit the state initial-dependent dynamical behaviors. By keeping the system parameters unchanged and changing the state initials, the trajectories of the memristive circuits and systems can asymptotically approach to different stable states, showing the state initial-dependent multistability $[2,3]$ or extreme multistability [4-7], i.e., coexisting multiple or infinitely many attractors. On the one hand, these coexisting multistable modes can provide more flexibility for information engineering applications [8-11]; on the other hand, it may also lead the application systems to abnormal working states [12]. These ungovernable problems pose a severe test for realizing the control of multistable modes. In addition, the dynamical behaviors of memristive circuits and systems are highly dependent on the state initials, but the state initials cannot be explicitly expressed in their state equations, which bring great obstacles in mechanical analyses of the state initial-dependent dynamical behaviors. Moreover, the memristive circuits and systems usually have line equilibrium set, plane equilibrium set, no equilibrium, or stable equilibrium, whose stabilities and induced dynamical behaviors are hard to be analyzed by using the traditional stability theory [12]. Therefore, accurate prediction, quantitative analysis, and physical control of such special phenomena have become an important research problem in the field of information science.

Traditional control strategies usually adopted nonfeedback control strategy to convert a multistable system to a mono-stable system [13-17] or adopted feedback control 
strategy to stabilize the system in a certain desired state $[13,18,19]$. But these control strategies cannot achieve the multistable control. To solve this problem, researchers proposed different dimensionality reduction modeling schemes based on the memristive circuit and system [20,21]. In [22], the concept of dimensionality reduction modeling was proposed, which modeled the memristive circuits with two physical quantities of flux and charge as main state variables, and the dimension of the obtained flux-charge model was lower than that of the traditional voltage-current model. Bao et al. [23] built the reduce-ordered flux-charge model of a two-memristor-based circuit and analyzed its dynamical characteristics via the voltage-current and fluxcharge models. Bao et al. [24] qualitatively pointed out that the flux-charge model of the memristive circuit can be equivalent to realize its dynamical behavior in the voltagecurrent model. However, in these early studies, the state initials of the memristive circuit were not explicitly expressed in the dimensionality reduction model [25], resulting in the information loss of state initials of the memristive circuit. Therefore, the established dimensionality reduction model could not reflect the multistability of the memristive circuit. In recent years, Corinto proposed an incremental flux-charge analysis method $[26,27]$ and applied it to the dimensionality reduction modeling of memristor-based cellular neural networks [28] and memristor-based oscillator array [29]. With this method, the state initials of the memristive circuit can be expressed as standalone system parameters in the flux-charge domain [12], which is conducive to the analyses and measurements of the state initial-dependent dynamical behaviors. Thereafter, this method was applied for reconstituting and analyzing extreme multistability of ideal memristor-based circuits $[12,20,30,31]$. On this basis, the incremental integral transformation method was proposed for the analyses of memristive systems [21,32]. Hereto, a complete set of dimensionality reduction reconstruction theory for ideal memristor-based circuits and systems was thereby formed.

It should be noted that, in the original literature, these two methods were called the flux-charge analysis method $[26,27]$ and the state variable mapping method [21], respectively. But the state variables of the dimensionality reduction model are actually expressed by the incremental integral of the original memristive circuit's and system's state variables, whose core idea is integral transformation. Therefore, in this paper, these two methods are called the incremental flux-charge analysis method and the incremental integral transformation method, respectively. By using these two methods, on the one hand, the implicit state initials in the original memristive circuit and system can be transformed into the explicitly state initial-related system parameters appearing in the dimensionality reduction model. On the other hand, the line or plane equilibrium set in the original memristive circuit and system can be converted into the certain equilibrium, which is beneficial to the elaboration of the dynamic mechanism. In short, the state initial-dependent dynamical behaviors of the original memristive circuit and system are transformed into the parameter-dependent dynamical behaviors of the dimensionality reduction model. In addition, synchronization, as one of the basic nonlinear phenomena, has received extensive attention in the field of basic theory and engineering applications [33]. In the nonmemristor-coupled system, the state initials have significant effect on the synchronization characteristics [34-36]. Based on the above dimensionality reduction methods, in the study of the synchronization on the memristor-coupled system [37], the synchronization effect of the state initial-related system parameters can be studied quantitatively after the state initials are expressed explicitly in the state equation.

The incremental flux-charge analysis method and the incremental integral transformation method realize the mapping transformation of the state variable domain by means of integral transformation and describe and analyze the multistability of the original memristive circuit and system based on the transformed state variable domain, which provides theoretical basis for the precise prediction, quantitative analysis, and physical control of such special phenomena. In this paper, the dimensionality reduction modeling and multistability reconstruction of the memristive circuit and system are summarized to help researchers fully understand the state initial-dependent multistability dimensionality reduction reconstruction strategy of the memristive circuit and system. Then, the reconstruction strategy is applied to the synchronization research of the memristor-coupled system to quantitatively study the influence of state initials on synchronization.

\section{Multistability of Memristive Circuit in the Voltage-Current Domain}

2.1. Multistability and Coexisting Multiple Attractors. Multistability $[13,38-41]$ is an inherent phenomenon of the nonlinear dynamical system, in which multiple attractors coexist with the change of state initials under the fixed system parameters. The term "multistability" first appeared in the study of visual perception [42]. Arecchi also found the coexistence attractors' phenomenon in electronic circuits [43] and gas lasers [44]. Later, a large number of theoretical and experimental studies have explored this special phenomenon in different systems [45-55]. In addition, in some special coupled systems [56, 57], the phenomenon of coexisting infinitely many attractors, i.e., extreme multistability [58-61], can also be observed.

In recent years, the hidden attractor [62-76], as a special class of newly defined attractor, has attracted extensive attention from researchers. The attractor that we usually say is also called the self-excited attractor, which is caused by the unstable equilibrium. Unlike the self-excited attractor $[67,77]$, the attraction basin with the hidden attractor does not intersect any equilibrium [78], and its existence increases the uncertainty of the system. When the system has a stable equilibrium [5] or no equilibrium [3, 79, 80], the induced multistability is called hidden multistability. Note that when the system has a stable equilibrium [5] and can produce dynamical behavior other than the point attractor, it can be confirmed that the system has hidden multistability. However, if the system has no equilibrium and can produce 
only one stable oscillation behavior, the system is a hidden system, but it does not have multistability [81].

It has been shown that the hidden attractor is sensitive to the state initial of the system. In the domain of stable equilibrium, the system trajectory will converge to the stable point, but under the special state initial, the system trajectory can form the stable chaotic attractor or periodic limit cycle. Figures 1(a) and 1(b), respectively, show the self-excited and hidden attractors generated by a novel Chua's circuit [67]. Figures 1(c) and 1(d), respectively, show the local plane projection of Figures 1(a) and 1(b), where the red dots are a pair of nonzero equilibria. According to the orbit of the attractor and the position relationship of the equilibrium in the figure, it can be seen that the attraction basin of the selfexcited attractor must cover the unstable equilibrium, while the attraction basin of the hidden attractor with the neighborhood of the stable equilibrium does not overlap. Therefore, the self-excited attractor and hidden attractor can be clearly distinguished according to the intersection relationship between the attraction basin and the equilibrium neighborhood in phase space.

In general, coexisting infinitely many attractors can be classified into the following four types:

(a) Different attractor types: hyperchaotic attractor, chaotic attractor, quasi-periodic limit cycle, periodic limit cycle, and stable point

(b) Different attractor topologies: the same type of attractor has completely different topologies, such as spiral and double-scroll attractors, scroll complete and incomplete attractors, and attractors with different dynamic amplitude

(c) Different number of limit cycles: limit cycles with different number of periods

(d) Different attractor positions: attractors are located in different phase space.

\subsection{The Difference between Multistability and Chaotic Initial} Sensitivity. Since Chua put forward the generalized concept of the memristive system [82], the circuit and system constructed by the memristor have received great attention. In the early memristive circuit [83-87], scholars found that the stability of the equilibrium was closely related to the state initial of the memristor, which meant that the coexistence of multiple attractors was easy to occur in the memristive circuit. Then, in the memristive circuit, Bao found that the state initial-dependent dynamical behavior was a special kind of multistability phenomenon, i.e., extreme multistability. And, in [88], Bao et al. explicitly proposed the extreme multistability in the memristive circuit for the first time, that is, in the memristive circuit with line equilibrium, there was a peculiar coexistence infinitely many attractor phenomenon, which relied on the internal state initial of the memristor. In particular, Jafari et al. [89] pointed out the difference between the state initial-dependent dynamical behavior (extreme multistability) of the memristive system and the chaotic initial sensitivity of the general nonlinear dynamical system. That is, for the general nonlinear dynamical system, the initial sensitivity of system trajectory was only a quantitative change, and the trajectories of the system starting from the different state initials would traversal in the corresponding attraction region along different trajectories, without changing the dynamical properties of the system. However, the extreme multistability of the memristive system was a qualitative change; the change of state initial could cause the trajectory of the memristive system to jump between the attraction domains of different dynamical behaviors. Therefore, the state initial-dependent multistability in the memristive circuit and the chaotic initial sensitivity in the general chaotic circuit are two completely different concepts.

2.3. Multistability in Memristive Circuit and System. Since physical accessibility of memristors has been reported [90], lots of investigations were carried out for various memristorbased application circuits and systems, including cellular nonlinear/neural network [91], spiking and bursting neuron circuit [92], active band-pass filter-based oscillating circuit [93], FitzHugh-Nagumo neuron circuit [94], recurrent neural network [95], hypogenetic jerk chaotic system $[21,96,97]$, and hyperchaotic autonomous system [98], from which rich dynamical behaviors have been manifested by theoretical studies, numerical simulations, and experimental measurements. The results showed that the stabilities of the memristive circuit and system, especially the ideal memristor-based nonlinear circuit and system, had a great relationship with the state initial of the memristor [88, 99]. Therefore, the coexisting infinitely many attractors appeared in such memristive circuit and system [12, 20, 100]. Under the fixed system parameters, the solution trajectories of the system can be represented by diverse stable states with the varied state initials, such as point, period, quasi-period, chaos, and hyperchaos [7, 98, 101, 102]. Such a special phenomenon is mostly relevant to no equilibrium $[103,104]$, limited number equilibria [105], or even infinitely many equilibria [6, 106]. Particularly, when the number of coexisting attractors tends to infinite, the phenomenon is called extreme multistability [39, 56, 89, 107-109].

In principle, the coexisting infinitely many attractors caused by extreme multistability generally has a complete smooth bifurcation route with respect to the state initial, and the bifurcation trajectories are gradual [110], such as perioddoubling bifurcation and Hopf bifurcation, as shown in Figure 2. It is important to emphasize that extreme multistability is not the same as coexisting infinitely many attractors. The aforesaid coexisting infinitely many attractors are commonly triggered in the memristive circuit and system with line or plane equilibrium set, entirely different from those generated from the offset-boostable flow by introducing an extra periodic signal [111-113] and also different from those generated from the attractor position offset caused by the state initial [114].

According to the definition of memristor $[1,82,115,116]$, researchers have proposed a variety of physical realizable memristor simulators with the characteristics of memristor ports [117], which can be mainly 


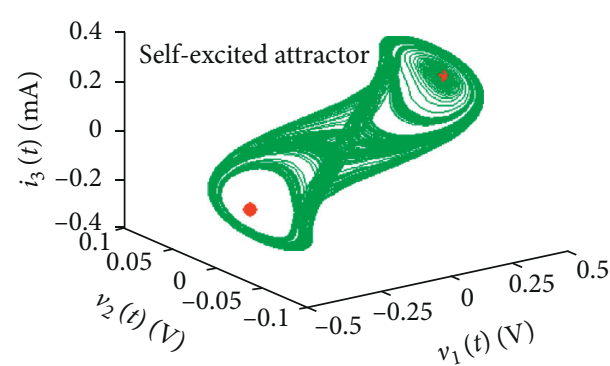

(a)

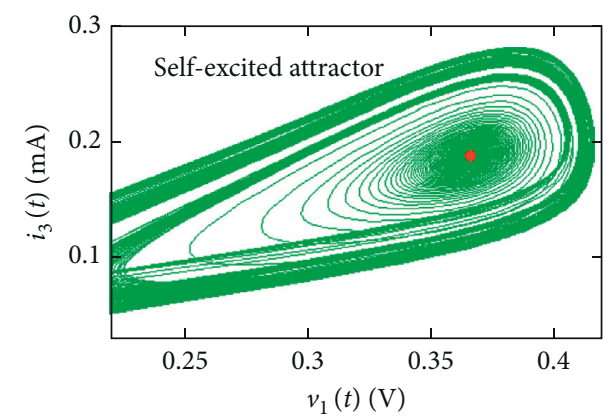

(c)

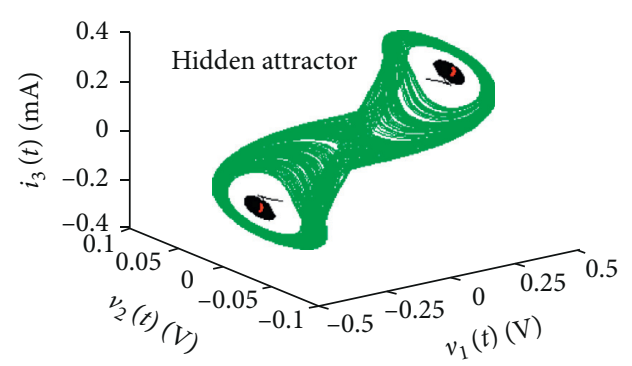

(b)

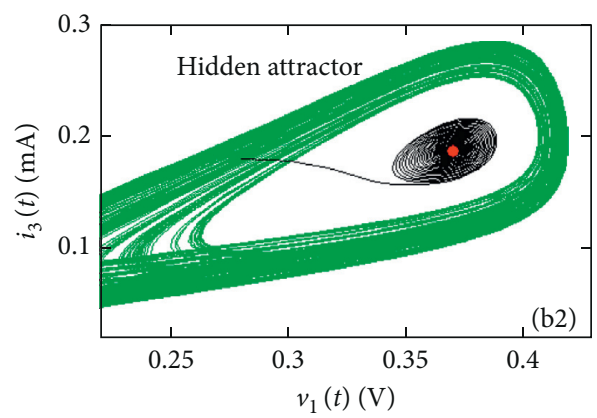

(d)

Figure 1: The generated attractors from a novel Chua's circuit (the red points are the nonzero equilibria): (a) self-excited attractor; (b) hidden attractor; (c) local plane projection of $a$; (d) local plane projection of $b$.

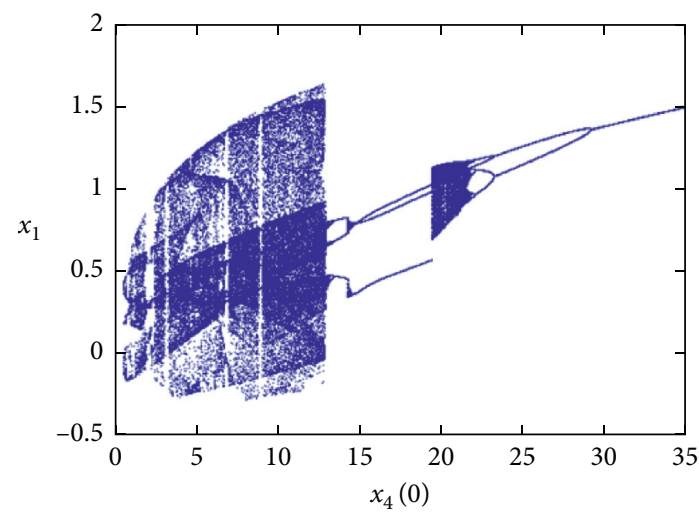

(a)

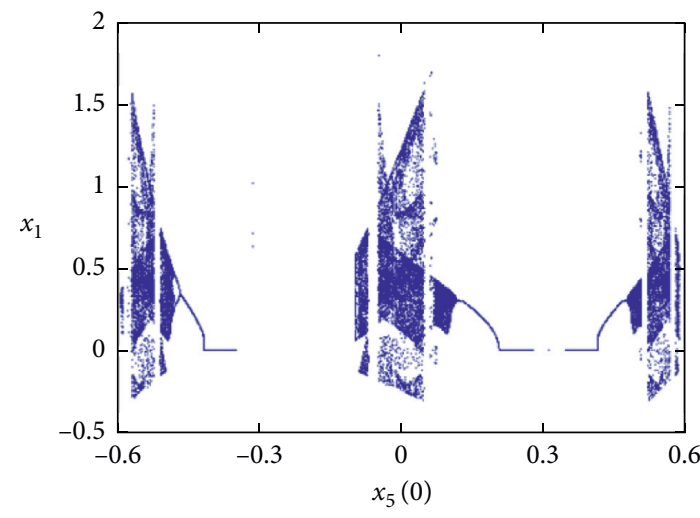

(b)

FIGURE 2: Extreme multistability: (a) with the variation of memristive initial $x_{4}(0)$; (b) with the variation of memristive initial $x_{5}(0)$.

divided into two categories: one is the ideal memristor or nonideal memristor based on the equivalent realization of operational amplifier and analog multiplier $[62,101,118,119]$; the other is the generalized memristor with diode bridge cascade RC, RL, or LC filters [120-124]. From the essential definition of the ideal memristor [116], it can be seen that the memristor is derived from the relationship between flux and charge [115]. The ideal memristor is usually divided into the charge-controlled memristor and flux-controlled memristor. Its voltage-current relation curve has the characteristic of typical italic " 8 " type pinched hysteresis loop, and the main characteristics are zero crossing $[82,116,125]$, double value [115], singular symmetry, tapering [116, 126], self-crossing type [127], and stability [128].

The nonideal memristor-based nonlinear circuit or system usually has certain equilibria [129], and their stability is not affected by the state initials. However, under the fixed system parameters, with the varied state initials, the system will produce the coexistence steady-state mode [2], namely, multistability. And, when a memristive circuit or system has a stable equilibrium or no equilibrium $[3,5]$, the system will produce hidden multistability.

The ideal memristor-based nonlinear circuit or system usually has infinitely many equilibria, and their positions and stabilities are related to the internal state initials of 
memristors, which indicates the extreme multistability of the memristive circuit or system. Bao et al. [88] proposed an ideal flux-controlled memristor-based Chua's circuit with line equilibrium set and revealed the state initial-dependent extreme multistability phenomenon of the memristive circuit. In [93], the ideal flux-controlled memristor was used to replace Chua's diode, and a memristive circuit with line equilibrium set was obtained, and the extreme multistability phenomenon of the circuit was studied. By introducing two ideal memristors into Chua's circuit, a memristive circuit with a plane equilibrium set was obtained in [130] and further revealed the extreme multistability phenomenon. By introducing an ideal flux-controlled memristor into a threedimensional hypogenertic jerk system, the paper [96] constructed a memristive system with four line equilibria sets, which could produce the extreme multistability phenomenon dependent on the state initial of the memristor and other state initials. Yuan et al. [107] designed a memristor-based multiscroll hyperchaotic system by introducing an ideal flux-controlled memristor and revealed its extreme multistability phenomenon. By introducing a microperturbation into the memristive circuit, a memristive circuit with no equilibrium was constructed, which could produce the phenomenon of hidden extreme multistability [131].

\section{Multistability Control Strategy}

3.1. Multistability Generic Control Strategy. Multistability has been reported in different scientific fields such as physics, chemistry, biology, and economy [13]. Because of its sensitive dependence on state initial, the multistability phenomenon can induce the system to switch between different coexisting states under the fixed system parameters, which provides great flexibility for the engineering application of the multistable system $[8,9,13,105,132-134]$. But, at the same time, it is easy to lead the application systems to abnormal working states, which puts forward a severe test to the multistable mode control strategy. For example, in the design of equipment with certain characteristics, it is necessary to avoid multistable or to stabilize it in the desired state, which will cause a lot of inconvenience in practical application. Therefore, it is necessary to control the multistable through appropriate control strategy.

In order to convert a multistable system to a mono-stable system, nonfeedback control strategy was usually adopted. In other words, by adding external disturbance to the system, such as the introduction of short pulse [13], a specific attractor could be selected in a multistable system to achieve multistability control. By introducing pseudoperiodic driving $[14,15]$ or harmonic disturbance $[16,17]$, the undesirable attractor types could be eliminated, and then, the system could be controlled in a certain stable state. In order to stabilize the system in a certain desired state, feedback control strategy was usually adopted [18], such as periodic driving [19] and time-delay feedback [13]. Yet these control strategies cannot achieve the multistable control. However, via the special constitutive relation of the memristor, some scholars have proposed appropriate multistability dimensionality reduction reconstitution strategies for specific types of the memristive circuit and system and realized the control of multistable modes. A brief introduction is given below.

\subsection{Multistability Dimensionality Reduction Reconstitution} Strategy. The multistability of the memristive circuit/system can provide more flexibility for the memristive circuit/ system to be applied in engineering application fields of image processing, signal encryption, and so on $[8,9,105,132-137]$. However, due to the sensitive dependence of the multistability on the state initial, there are two main problems when the traditional analysis method is used to analyze the multistable mode of the memristive circuit/ system. On the one hand, the dynamical behaviors of the multistable circuit/system are highly dependent on the state initials, but the state initials cannot be expressed explicitly in the state equation of the multistable circuit/system, which makes it impossible to quantitatively analyze the state initialdependent dynamical behavior of the memristive circuit/ system. On the other hand, since the memristive circuit/ system usually has line equilibrium set, plane equilibrium set, space equilibrium set, or no equilibrium, when we use the traditional analysis method to analyze the dynamical behavior, it is very difficult to correctly judge whether the equilibrium of the system is stable or not, or cannot analyze the system equilibrium, which makes it impossible to quantitatively describe the internal mechanism of multistability. These problems make it difficult to accurately predict, quantitatively analyze, and physically control the state initial-dependent dynamical behaviors.

Therefore, in the process of analyzing the multistability of the memristive circuit and system, in order to solve these problems, researchers proposed different dimensionality reduction modeling schemes based on the memristive circuit [20] and memristive system [21]. In fact, a prototype of dimensionality reduction modeling had been developed in the earlier literature [22-24]. In [22], the concept of dimensionality reduction modeling was proposed, which modeled the memristive circuits with two physical quantities of flux and charge as main state variables, and the dimension of the obtained flux-charge model was lower than that of the traditional voltage-current model. Bao et al. [23] built the reduce-ordered flux-charge model of a two-memristorbased memristive circuit and analyzed its dynamical characteristics via the voltage-current and flux-charge models. Bao et al. [24] qualitatively pointed out that the flux-charge model of the memristive circuit could be equivalent to realize its dynamical behavior in the voltage-current model. However, in these early studies, the state initials of the memristive circuits were not explicitly expressed in the dimensionality reduction model [25], resulting in the information loss of state initials of the memristive circuit. Therefore, the established dimensionality reduction model could not reflect the original multistability of the memristive circuits and systems.

In recent years, Corinto proposed an incremental fluxcharge analysis method [26], the method was based on the 
Kirchhoff flux and charge law and the constitutive relation of the circuit element under the incremental flux and incremental charge. Compared with the circuit equation in the voltage-current domain, the circuit equation in the fluxcharge domain established by this method had a simpler equation structure, which could simplify the complexity of dynamical analysis and clearly understand the influence of state initial. To further demonstrate the effectiveness of the method, Corinto applied the method to the analysis of Hopf bifurcation and period-doubling cascade induced by state initial [27]. Subsequently, more scholars applied it to the study of complex memristive circuits such as memristorbased cellular neural network [28] and memristor-based oscillator array [29]. Then, Chen clearly proposed that this method could represent the state initials of all dynamical elements in the circuit as standalone state initial-related system parameters [12], which was conducive to the analysis and measurement of the state initial-dependent dynamical behaviors in the memristive circuit. Moreover, this method was applied to the reconstruction and analysis of extreme multistability for the ideal memristor-based circuit $[12,20,30,31]$. And, on this basis, the incremental integral transformation method for the memristive system was proposed [21, 32], forming a complete set of dimensionality reduction reconstruction theory for ideal memristor-based circuits and systems. That is, firstly, the integral transformations on all state variables of the original memristive circuit/system are carried out (note that all terms in the system equation must be integrable). Then, the dimensionality reduction modeling is implemented by using the nondynamic property [22] of the memristor in the fluxcharge domain. Then, based on the dimensionality reduction model, the state initial-dependent dynamical behaviors of the original memristive circuit/system are reconstructed and analyzed.

\section{Flux-Charge Constitutive Relation of Memristor}

In the 1970s, Chua proposed the fourth basic circuit element, memristor, to characterize the relationship between flux and charge [115] and deduced the existence the memristor from the symmetry of circuit variables and the characteristics of the electromagnetic field, as shown in Figure 3. As can be seen from Figure 3, there are four basic physical quantities in the circuit: current $i$, voltage $v$, charge $q$, and flux $\varphi$. There are six mathematical relations among them; among which the relations between current and charge and voltage and flux are as follows:

$$
\begin{aligned}
& q(t)=\int_{-\infty}^{t} i(\xi) \mathrm{d} \xi \\
& \varphi(t)=\int_{-\infty}^{t} v(\xi) \mathrm{d} \xi
\end{aligned}
$$

Equations (1a) and (1b), respectively, represent that charge is the integral of current with respect to time and flux is the integral of voltage with respect to time. According to

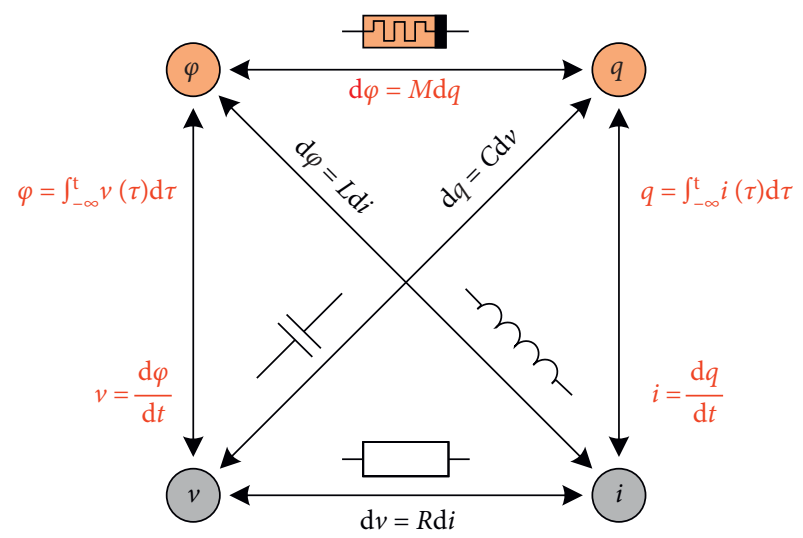

Figure 3: Circuit basic variables and four basic elements.

the incremental flux-charge analysis method, Corinto and Forti [26] gave the definition of incremental charge and incremental flux for any $t \geq t_{0} \quad\left(-\infty<t_{0}<\infty\right)$, i.e., $q\left(t ; t_{0}\right)=\int_{t_{0}}^{t} i(\xi) \mathrm{d} \xi, \varphi\left(t ; t_{0}\right)=\int_{t_{0}}^{t} v(\xi) \mathrm{d} \xi$, and equation $(1)$ can be further written as

$$
q(t)=\int_{-\infty}^{t} i(\xi) \mathrm{d} \xi=\int_{-\infty}^{t_{0}} i(\xi) \mathrm{d} \xi+\int_{t_{0}}^{t} i(\xi) \mathrm{d} \xi=q\left(t_{0}\right)+q\left(t ; t_{0}\right),
$$

$\varphi(t)=\int_{-\infty}^{t} v(\xi) \mathrm{d} \xi=\int_{-\infty}^{t_{0}} v(\xi) \mathrm{d} \xi+\int_{t_{0}}^{t} v(\xi) \mathrm{d} \xi=\varphi\left(t_{0}\right)+\varphi\left(t ; t_{0}\right)$.

It is well known that charge and flux are internal state variables of the memristor in the voltage-current domain, and the internal state initial represents the memory property of the memristor. However, its state initial cannot appear explicitly in the state equation, so it is naturally impossible to assign its value accurately. Therefore, the memory of the memristor cannot be simulated effectively in the voltagecurrent domain. Compared with the two basic physical quantities of voltage and current, flux and charge can better represent the basic physical properties of circuit elements [138], which provide a theoretical basis for exploring the intrinsic properties of circuit elements. To this end, it is assumed that the voltage $v(t)$ and the current $i(t)$ on the memristor adopt the associated reference direction, and two different types of memristors are selected to build their fluxcharge constitutive relation.

4.1. Flux-Charge Constitutive Relation of Charge-Controlled Memristor. For the charge-controlled memristor in Figure 4(a), the voltage-current relationship between the current $i(t)$ flowing through it and the voltage $v(t)$ at both ends of it in the voltage-current domain can be described as

$$
v(t)=M\left(q_{M}\right) i(t)
$$

where the memristive function $M\left(q_{M}\right)$ is the nonlinear function about charge $q_{M}(t)$ and has the same dimension as resistance; the unit is ohms $(\Omega)$. In this voltage-current model, $q_{M}(t)$ is the internal state variable of the memristor, 


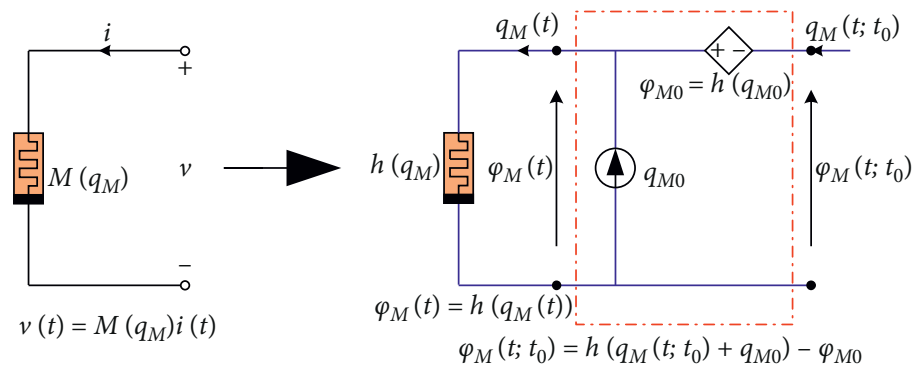

(a)

(b)

Figure 4: Charge-controlled memristor. The constitutive relation transformation from the voltage-current domain to the flux-charge domain: (a) voltage-current constitutive relation; (b) flux-charge constitutive relation.

and its state initial $q_{M}\left(t_{0}\right)$ represents the memory property of the memristor.

Compared with the voltage-current domain, we take charge and flux as state variables in the flux-charge domain; take the integral from $-\infty$ to $t$ for both sides of equation (3), and combine with equation (1) to obtain the flux-charge constitutive relation of the charge-controlled memristor in the flux-charge domain as follows:

$$
\varphi_{M}(t)=\int_{-\infty}^{t} v(\xi) \mathrm{d} \xi=\int_{-\infty}^{t} M\left(q_{M}\right) i(\xi) \mathrm{d} \xi=h\left(q_{M}(t)\right)
$$

where the function $h(\bullet)$ is the nonlinear function about $q_{M}(t)$.

According to equations (2a) and (2b), equation (4) can be further rewritten as

$$
\varphi_{M}\left(t ; t_{0}\right)=h\left(q_{M}\left(t ; t_{0}\right)+q_{M 0}\right)-\varphi_{M 0}
$$

where $\varphi_{M 0}=\varphi_{M}\left(t_{0}\right)$ and $q_{M 0}=q_{M}\left(t_{0}\right)$; equation (5) describes the memristor as a special nonlinear element whose memory is explicitly shown by the internal state initial $q_{M 0}$ [27], i.e., the internal state initial of the memristor can be explicitly expressed in the flux-charge domain, which is conducive to the quantitative analysis of state initial-dependent dynamical behavior. By comparing equation (5) with equation (3), it is not difficult to conclude that the state variable in the fluxcharge domain is expressed by the incremental integral of the state variable in the voltage-current domain, and its state initial is zero, i.e., when $t=t_{0}, \varphi_{M}\left(t_{0} ; t_{0}\right)=q_{M}\left(t_{0} ; t_{0}\right)=0$. Figure 4 visually shows the transformation of the chargecontrolled memristor from voltage-current constitutive relation to flux-charge constitutive relation.

\subsection{Flux-Charge Constitutive Relation of Flux-Controlled} Memristor. For the flux-controlled memristor in Figure 5(a), the voltage-current relationship between the current flowing through it and the voltage at both ends of it in the voltage-current domain can be described as

$$
i(t)=W\left(\varphi_{W}\right) v(t)
$$

where $\varphi_{W}$ is the internal state variable of the memristor and $W\left(\varphi_{W}\right)$ is the memductance function.
Similar to the charge-controlled memristor, charge and flux are used as state variables in the flux-charge domain. The integral of both sides of equation (6) from $-\infty$ to $t$ is taken, and the flux-charge constitutive relation of the fluxcontrolled memristor in the flux-charge domain is obtained by combining with equation (1) as follows:

$$
q_{W}(t)=\int_{-\infty}^{t} i(\xi) \mathrm{d} \xi=\int_{-\infty}^{t} W\left(\varphi_{W}\right) v(\xi) \mathrm{d} \xi=f\left(\varphi_{W}(t)\right)
$$

where the function $f(\bullet)$ is the nonlinear function about $\varphi_{W}(t)$.Further, equation (7) can be written as

$$
q_{W}\left(t ; t_{0}\right)=f\left(\varphi_{W}\left(t ; t_{0}\right)+\varphi_{W 0}\right)-q_{W 0},
$$

where $\varphi_{W 0}=\varphi_{W}\left(t_{0}\right)$ and $q_{w 0}=q_{W}\left(t_{0}\right)$. Similarly, the fluxcontrolled memristor can also be expressed as a special nonlinear element in the flux-charge domain, and its memory is reflected by the internal state initial $\varphi_{W 0}$; and, when $t=t_{0}, \varphi_{W}\left(t_{0} ; t_{0}\right)=q_{W}\left(t_{0} ; t_{0}\right)=0$. Figure $5(\mathrm{~b})$ shows the flux-charge constitutive relation of the flux-controlled memristor.

\section{Incremental Flux-Charge Analysis Method for Memristive Circuit}

Bao et al. [22] pointed out that, in the voltage-current model, the memristor was a dynamic element, resulting in an increase in the order of the circuit equation. In the flux-charge model, the memristor was a nondynamic element, so the order of the circuit remains the same. Therefore, for the memristive circuit, when flux and charge are taken as state variables rather than voltage and current [25, 139], the memristor is described as a nondynamic element, which can reduce the dimension of the established mathematical model $[12,20,30]$, from which the term "dimensionality reduction" is derived. It should be noted that the flux-charge model and the voltage-current model are different from each other in their algebraic equations, but they are equivalent representations in nonlinear dynamical behaviors. And, the implicit state initials of all dynamic components in the voltage-current model can be expressed as the explicit initial-related system parameters in the flux-charge model, which is convenient to realize the mechanism explanation of state initial-dependent dynamical behavior in the 


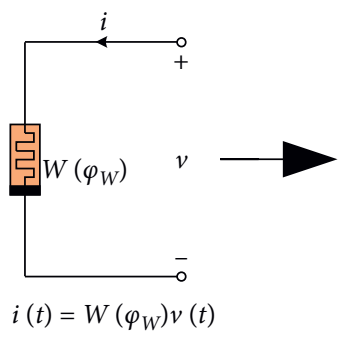

(a)

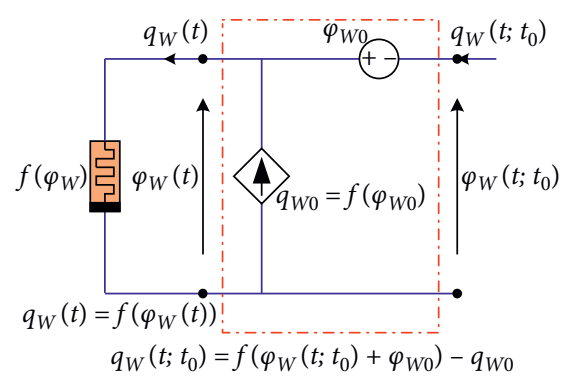

(b)

Figure 5: Flux-controlled memristor: the constitutive relation transformation from the voltage-current domain to the flux-charge domain; (a) voltage-current constitutive relation; (b) flux-charge constitutive relation.

memristive circuit, namely, to realize the multistability reconstitution [30]. In addition, dimensionality reduction modeling can reduce the complexity of quantitative analysis and numerical simulation, which has certain theoretical significance and engineering application value.

Bao et al. [130] proposed a two-memristor-based Chua's circuit, as shown in Figure 6, and revealed its state initialdependent extreme multistability phenomenon. Based on the memristive circuit, Chen adopted the incremental fluxcharge analysis method [31] and obtained the dimensionality reduction model in the flux-charge domain, which not only solved the special dynamic characteristics problem of the circuit which could not be quantitatively explained in the voltage-current domain but also made the system model simpler and more conducive to the analysis of its dynamical formation mechanism.

To be specific, this article mainly solved the following five problems:

(a) The 5-order dynamic circuit of the original system was described by a 3 -dimensional system model, and the dimensionality reduction modeling was realized. The two-memristor-based Chua's circuit [130] in the voltage-current domain was

$$
\left\{\begin{array}{l}
\frac{\mathrm{d} V_{1}}{\mathrm{~d} t}=-\frac{1}{R C_{1}}\left(V_{1}-V_{2}\right)+\frac{1}{R_{\mathrm{b}} C_{1}}\left(1-g_{1} V_{4}^{2}\right) V_{1}, \\
\frac{\mathrm{d} V_{2}}{\mathrm{~d} t}=-\frac{k}{R C_{2}}\left(V_{1}-V_{2}\right)+\frac{k}{R_{\mathrm{d}} C_{2}}\left(1-g_{2} V_{5}^{2}\right) V_{2}-\frac{2 k+1}{(k+1) R_{1} C_{2}} V_{3}, \\
\frac{\mathrm{d} V_{3}}{\mathrm{~d} t}=-\frac{k+1}{R C_{3}}\left(V_{1}-V_{2}\right)+\frac{k+1}{R_{\mathrm{d}} C_{3}}\left(1-g_{2} V_{5}^{2}\right) V_{2}-\frac{2}{R_{1} C_{3}} V_{3}, \\
\frac{\mathrm{d} V_{4}}{\mathrm{~d} t}=-\frac{1}{R_{\mathrm{a}} C_{4}} V_{1}, \\
\frac{\mathrm{d} V_{5}}{\mathrm{~d} t}=-\frac{1}{R_{\mathrm{c}} C_{5}} V_{2} .
\end{array}\right.
$$

Its dimensionality reduction model [31] in the flux-

charge domain was

$$
\left\{\begin{array}{l}
C_{1} \frac{\mathrm{d} \varphi_{1}\left(t ; t_{0}\right)}{\mathrm{d} t}=\frac{1}{R}\left(-\varphi_{1}\left(t ; t_{0}\right)+\varphi_{2}\left(t ; t_{0}\right)\right)-q_{4}\left(t ; t_{0}\right)+C_{1} V_{1}\left(t_{0}\right), \\
C_{2} \frac{\mathrm{d} \varphi_{2}\left(t ; t_{0}\right)}{\mathrm{d} t}=\frac{k}{R}\left(-\varphi_{1}\left(t ; t_{0}\right)+\varphi_{2}\left(t ; t_{0}\right)\right)+k q_{5}\left(t ; t_{0}\right)-\frac{2 k+1}{(k+1) R_{1}} \varphi_{3}\left(t ; t_{0}\right)+C_{2} V_{2}\left(t_{0}\right), \\
C_{3} \frac{\mathrm{d} \varphi_{3}\left(t ; t_{0}\right)}{\mathrm{d} t}=\frac{k+1}{R}\left(-\varphi_{1}\left(t ; t_{0}\right)+\varphi_{2}\left(t ; t_{0}\right)\right)+(k+1) q_{5}\left(t ; t_{0}\right)-\frac{2}{R_{1}} \varphi_{3}\left(t ; t_{0}\right)+C_{3} V_{3}\left(t_{0}\right),
\end{array}\right.
$$




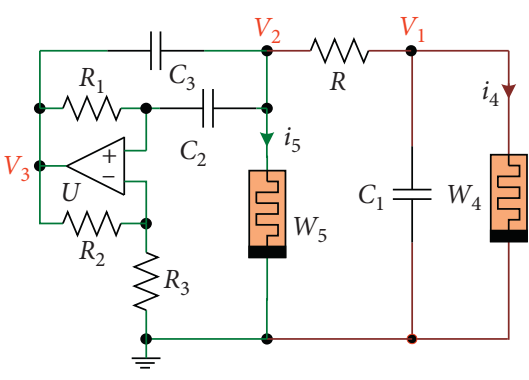

(a)

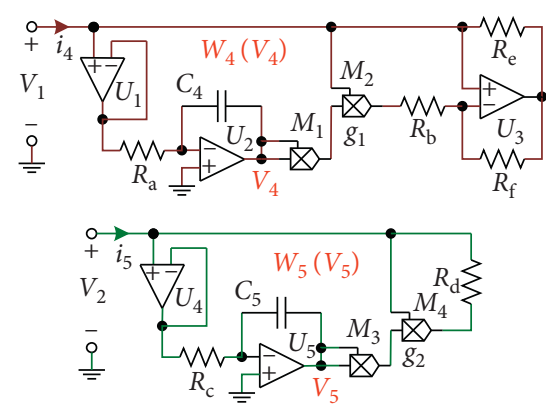

(b)

Figure 6: Two-memristor-based Chua's circuit: (a) circuit schematic of active BPF-based memristive Chua's circuit; (b) equivalent circuit for the memristors $W_{4}$ and $W_{5}$.

where

$$
\begin{aligned}
& q_{4}\left(t ; t_{0}\right)=-\frac{\varphi_{1}\left(t ; t_{0}\right)}{R_{\mathrm{b}}}\left[1-\frac{g_{1} \varphi_{1}^{2}\left(t ; t_{0}\right)}{3\left(R_{\mathrm{a}} C_{4}\right)^{2}}\right]-\frac{\varphi_{1}\left(t ; t_{0}\right)}{R_{\mathrm{b}}}\left[-g_{1} V_{4}^{2}\left(t_{0}\right)+\frac{g_{1} V_{4}\left(t_{0}\right)}{R_{\mathrm{a}} C_{4}} \varphi_{1}\left(t ; t_{0}\right)\right], \\
& q_{5}\left(t ; t_{0}\right)=\frac{\varphi_{2}\left(t ; t_{0}\right)}{R_{\mathrm{d}}}\left[1-\frac{g_{2} \varphi_{2}^{2}\left(t ; t_{0}\right)}{3\left(R_{\mathrm{c}} C_{5}\right)^{2}}\right]+\frac{\varphi_{2}\left(t ; t_{0}\right)}{R_{\mathrm{d}}}\left[-g_{2} V_{5}^{2}\left(t_{0}\right)+\frac{g_{2} V_{5}\left(t_{0}\right)}{R_{\mathrm{c}} C_{5}} \varphi_{2}\left(t ; t_{0}\right)\right] .
\end{aligned}
$$

(b) Converted the plane equilibrium set of the original memristive circuit to three or five determined equilibria.

Original memristive circuit (9) had a plane equilibrium set $P=\left\{\left(V_{1}, \quad V_{2}, \quad V_{3}, \quad V_{4}, \quad V_{5}\right) \mid\right.$ $\left.V_{1}=V_{2}=V_{3}=0 \mathrm{~V}, V_{4}=\mu \mathrm{V}, V_{5}=\eta \mathrm{V}\right\}$, which led to two critical stable zero eigenvalues at the equilibrium set. Therefore, it was impossible to accurately determine the stability of equilibrium set, resulting in local inconsistency between the stability interval divided by the nonzero eigenvalues and the actual observed dynamical behavior. In flux-charge dimensionality reduction model (10), the plane equilibrium set was transformed into three or five determinate equilibria which were related to the initial-related system parameter $V_{i}\left(t_{0}\right)(i=1,2,3,4$, and 5), which eliminated the ill-posed zero eigenvalues of the original memristive circuit. According to the evolution characteristics of the determined equilibria with $V_{i}\left(t_{0}\right)$, the theoretical explanation of the inconsistency between the stability interval of the equilibrium set and the dynamical behavior of the original memristive circuit was given, and the state initial-dependent dynamical mechanism of the original memristive circuit was quantitatively expounded.

(c) Reasonable reasons for the significant change of dynamical behavior under the change of small state initial were expounded.

The state initial $V_{1}\left(t_{0}\right)$ of original memristive circuit (9) was fine-tuned from $10^{-9}$ to $-10^{-9}$ (that is, the initial-related system parameter of dimensionality reduction model (10) was fine-tuned from $10^{-9}$ to $\left.-10^{-9}\right)$, and the dynamical behavior changed greatly. This phenomenon could not be reasonably explained in the voltage-current domain, but after dimensionality reduction modeling, it could be explained in the flux-charge domain according to the symmetry of dimensionality reduction model (10).

(d) Extreme multistability reconstitution was implemented.

The implicit state initial $V_{i}\left(t_{0}\right)$ of system (9) was explicitly expressed in dimensionality reduction model (10) as the initial-related system parameter. When the state initial of system (10) was set as $(0,0$, 0 ), the kinetic map shown in [31] had the same dynamical behavior as the attraction basin shown in [130], intuitively illustrated dimensionality reduction model (10), perfectly reconstructed the state initial-dependent dynamical behavior of original memristive circuit (9), and realized the extreme multistability reconstitution.

(e) In the hardware circuit of the flux-charge model, the multistable mode control of the memristive circuit was realized by changing the initial-related system parameters.

\section{Incremental Integral Transformation Method for Memristive System}

On the basis of the incremental flux-charge analysis method, for the ideal memristor-based system, the incremental 
integral transformation method was proposed in [21] to obtain an equivalent dimensionality reduction model. From the new state variable domain, the state initial-dependent extreme multistability of the original memristive system was studied quantitatively, so as to realize the reconstitution of the extreme multistability, and then, the theoretical basis of the dimensionality reduction reconstitution of the memristive system was given [110]. However, it should be noted that this method only applies to the simple ideal memristorbased system with only memristor nonlinear terms. But for the complex memristive system with other nonlinear terms besides the memristor nonlinear term [32], because it is difficult to obtain an explicit expression of the time integral of complex nonlinear terms, it is necessary to find appropriate intermediate variables and variable substitution to achieve the purpose of equivalent dimensionality reduction modeling. For this reason, the hybrid incremental integral transformation method was proposed in [32].

\subsection{Incremental Integral Transformation Method for Simple} Memristive System. For the simple ideal memristor-based system with only the memristor nonlinear term, the incremental integral transformation method is used to realize dimensionality reduction modeling and multistability reconstitution. Taking the memristive hyperjerk system as an example, the system has only one memristor nonlinear term with smooth hyperbolic tangent memductance [140], and its mathematical model is

$$
\left\{\begin{array}{l}
\dot{x}_{1}=x_{2}, \\
\dot{x}_{2}=x_{3}, \\
\dot{x}_{3}=x_{4}, \\
\dot{x}_{4}=\tanh \left(x_{1}\right) x_{2}-x_{3}-0.5 x_{4} .
\end{array}\right.
$$

According to the incremental integral transformation method [21], the dimensionality reduction model can be obtained as

$$
\left\{\begin{array}{l}
\dot{X}_{2}=X_{3}+\delta_{2}, \\
\dot{X}_{3}=X_{4}+\delta_{3}, \\
\dot{X}_{4}=-X_{3}-0.5 X_{4}+\ln \cosh \left(X_{2}+\delta_{1}\right)-\ln \cosh \left(\delta_{1}\right)+\delta_{4} .
\end{array}\right.
$$

The corresponding equilibrium is transformed from the line equilibrium set to two determined equilibria, and the state variables between the dimensionality reduction model and the original system have the following corresponding relationship:

$$
\begin{aligned}
& x_{1}=X_{2}+\delta_{1}, \\
& x_{2}=X_{3}+\delta_{2}, \\
& x_{3}=X_{4}+\delta_{3}, \\
& x_{4}=\dot{X}_{4} .
\end{aligned}
$$

It should be noted that the system parameter $\delta_{i}(i=1,2$, 3 , and 4 ) of the dimensionality reduction model represents the state initial $x_{i}(0)(i=1,2,3$, and 4$)$ of the original memristive system. Similar to the above incremental fluxcharge analysis method, when the initial is set to $(0,0,0)$, based on the dimensionality reduction model, the state initial-dependent extreme multistability reconstitution of the original memristive system can be realized.

6.2. Hybrid Incremental Integral Transformation Method for Complex Memristive System. For the ideal memristor-based system with other nonlinear terms besides the memristor nonlinear term, the hybrid incremental integral transformation method was proposed in [32], which successfully solved the dimensionality reduction modeling and multistability reconstitution problems of the memristive system with complex nonmemristor cubic nonlinear terms.

To be specific, this article mainly solved the following three problems:

(a) By introducing a new intermediate variable, the problem that the nonmemristor cubic nonlinear integral term could not be expressed by a simple relation was eliminated.

The mathematical model of a four-dimensional complex memristive system with a nonmemristor cubic nonlinear term [32] is described as

$$
\left\{\begin{array}{l}
\dot{x}_{1}=x_{2}, \\
\dot{x}_{2}=\left(1-x_{4}\right) x_{3}, \\
\dot{x}_{3}=x_{1}-a x_{2}-x_{3}-b x_{1}^{3}, \\
\dot{x}_{4}=-x_{3} .
\end{array}\right.
$$

Incremental integral transformation method [21] was adopted to carry out integral transformation on the system. By introducing an intermediate variable $W=b \int_{0}^{t} x_{1}^{3} \mathrm{~d} \tau$, the problem that this integral term could not be expressed as a simple relational expression was eliminated, and a four-dimensional intermediate transformation system with the same dimension as the original system was obtained, that is,

$$
\left\{\begin{array}{l}
\dot{X}_{1}=X_{2}+\delta_{1} \\
\dot{X}_{2}=0.5 X_{3}^{2}+\left(1-\delta_{4}\right) X_{3}+\delta_{2}, \\
\dot{X}_{3}=X_{1}-a X_{2}-X_{3}-W+\delta_{3} \\
\dot{W}=b\left(X_{2}+\delta_{1}\right)^{3}
\end{array}\right.
$$

(b) The variable substitution method was used to eliminate the divergence of state variables in the intermediate transformation system, and then, the dimensionality reduction modeling was realized.

Through variable substitution $Y_{1}=X_{1}-W, Y_{2}=X_{2}$, and $Y_{3}=X_{3}$, the divergence problem of state variables $X_{1}$ and $W$ was eliminated, and the equivalent three-dimensional dimensionality reduction model of the system was obtained, i.e.,

$$
\left\{\begin{array}{l}
\dot{Y}_{1}=Y_{2}+\delta_{1}-b\left(Y_{2}+\delta_{1}\right)^{3} \\
\dot{Y}_{2}=0.5 Y_{3}^{2}+\left(1-\delta_{4}\right) Y_{3}+\delta_{2} \\
\dot{Y}_{3}=Y_{1}-a Y_{2}-Y_{3}+\delta_{3} .
\end{array}\right.
$$


(c) The extreme multistability reconstitution of the memristive system with other nonlinear terms besides memristor nonlinear terms was realized.

The three line equilibria sets of the original system were transformed into six determinated equilibria, and the ill zero eigenvalue of the original system was eliminated. And, the state initial $x_{i}\left(t_{0}\right)(i=1,2,3$, and $4)$, as the initial-related system parameter $\delta_{i}(i=1,2$, 3 , and 4), was explicitly expressed in the dimensionality reduction model. When $Y_{1}(0), Y_{2}(0)$, and $Y_{3}(0)$ were set to 0 , dimensionality reduction system (17) could reconstruct the extreme multistability of original system (15).

6.3. Hidden Extreme Multistability Reconstitution. Different from the memristive system mentioned above, the nonautonomous FitzHugh-Nagumo (FHN) neuronal circuit was used to solve the problem of critical stability (i.e., hidden attractors) of the system [141]. By using dimensionality reduction modeling, it was proved that the attractors generated by the system were indeed hidden [70, 141]. This article mainly solved the following four problems: (a) It made up for the gap that the nonautonomous memristive circuit produced extreme multistability. A memristor with a smooth hyperbolic tangent nonlinear memductance was used to replace the nonsmooth piecewise linear memductance in the FHN neuron circuit in [94]; a nonautonomous memristive FHN neuron model that could produce extreme multistability was obtained.

(b) By using the incremental integral transformation method, the original 3-dimensional system was transformed into 2-dimensional dimensionality reduction. The nonautonomous memristive FHN neuron model is described as

$$
\left\{\begin{array}{l}
\dot{x}_{1}=x_{2}+0.5\left(1-\tanh x_{3}\right) x_{1}+1.8 \cos (\tau), \\
\dot{x}_{2}=-x_{1}-x_{2}, \\
\dot{x}_{3}=-x_{1} .
\end{array}\right.
$$

After the incremental integral transformation, the model of dimensionality reduction was obtained:

$$
\left\{\begin{array}{l}
\dot{X}_{1}=X_{2}+0.5 X_{1}+0.5 \ln \cosh \left(X_{1}-\delta_{3}\right)+1.8 \sin (\tau)+\delta_{1}-0.5 \ln \cosh \left(\delta_{3}\right), \\
\dot{X}_{2}=-X_{1}-X_{2}+\delta_{2} .
\end{array}\right.
$$

(c) The critical stability of the original system was transformed into the deterministic stability of the dimensionality reduction model.

The original system contained the nonautonomous term, which caused the system's equilibrium to change alternately between stable line equilibrium set and no equilibrium with time. The attractor generated by no equilibrium was hidden. However, due to the existence of zero eigenvalue, the line equilibrium set had critical stability, so it was impossible to determine whether the system produced hidden attractor. After dimensionality reduction modeling, no equilibrium and zero eigenvalue were eliminated, and the dimensionality reduction model only had certain equilibria which changed with time and were always stable, thus the equilibria had certain stability; it was proved that the attractors generated by the original system were indeed hidden.

(d) The hidden extreme multistability reconstitution of the nonautonomous memristive system was realized.

\section{Synchronization Application of Memristor- Coupled System}

Because of the nano-sized property, memristors are used to mimic biological neuronal synapses [142-145], which play important roles in the process of information transmission among the coupled neurons [146-149]. And, various memristor-coupled systems are studied, such as memristorcoupled Hindmarsh-Rose neurons [150] and memristorcoupled Hopfield neural network [151, 152].

It is all known that abundant collective behaviors appear in the actual neural system due to the interactions in neurons $[153,154]$; among them, synchronization is the outstanding collective features in neuroscience [155-157], which is regarded as one of the mechanisms to propagate and to code information in brain $[158,159]$. However, there are different kinds of brain disorder diseases, such as Alzheimer's, epilepsy, Parkinson's, and schizophrenia, which are involved with the abnormal activities of synchronization [160]. Therefore, neuron synchrony is a fundamental topic in neuroscience.

Different from the traditional nonlinear elements, the memristor is a special nonlinear element with internal state variables [1]. Therefore, using the memristor to couple the nonlinear system can easily generate special synchronization behaviors that depend on the initials of the memristor, which is completely different from the general nonlinear coupling system $[161,162]$. In the general nonlinear coupled system, as long as the coupling strength is large enough, the master system and slave system starting from any state initials will always asymptotically achieve complete synchronization [163]. Naturally, in the nonmemristor-coupled system, some scholars have analyzed the initial influence on synchronization from the qualitative point of view and 


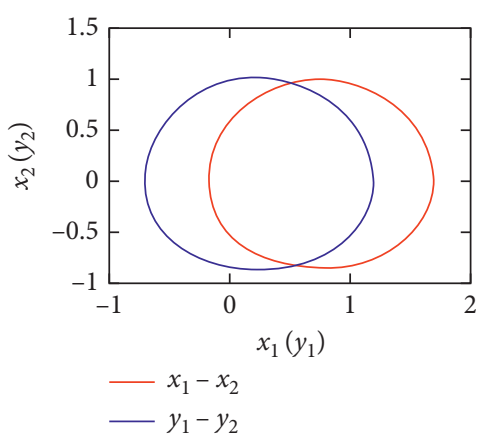

(a)

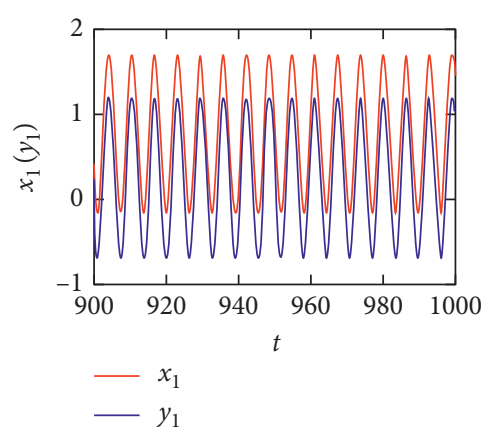

(b)

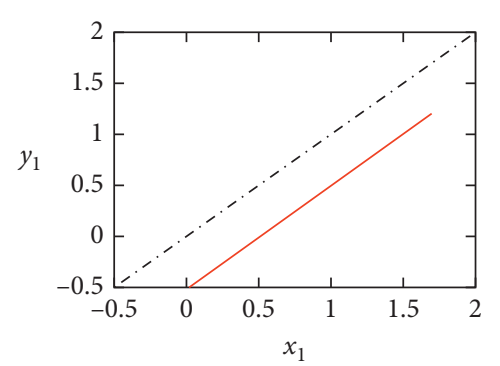

(c)

Figure 7: Periodic synchronizations with parallel offsets between the master system and slave system.

found that the synchronization stability depended on the state initial setting to some extent [35]. For memristorcoupled systems, various synchronization research studies have attracted important attention, and different influencing factors on synchronization were proposed. For examples, in [164], the effect of coupling strength on synchronization transition was investigated. In [165], the influence of coupling intensity and induction coefficient on phase synchronization was discussed. In [166], the effect of electromagnetic parameters on synchronization was studied. In [94], the effect of the coupling memristor parameter on synchronization was given. In $[147,167,168]$, the robust analysis approach to asymptotic finite-time synchronization and interval matrix method of global exponential synchronization were proposed for investigations of the delayed memristive neural networks.

However, the dynamical effects of the state initials on synchronization in the memristor-coupled systems were rarely concerned in the published literatures [37, 169, 170]. The first reason is that the state initials are implicit parameters and cannot be expressed explicitly in the state equations. Secondly, such a result makes many researchers question it, because according to our previous understanding of synchronization, state initials are irrelevant to synchronization behavior $[161,162,171]$; however, now, it is said that state initials have influence on synchronization behavior, and their influence cannot be ignored; most scholars are doubtful about this conclusion. At the same time, if the conclusion is presented only by numerical analysis and other qualitative means, its credibility is undoubtedly not enough.

Interestingly, these problems can be solved by simplifying the mathematical models via using appropriate state variables or applying reasonable approximation and simplification [21, 37, 172, 173]. In [173], the initial effects on synchronization for the memristor-coupled system were quantitatively analyzed by the incremental flux-charge analysis method. Due to the inherent state initial mismatches between the two identical coupling systems, the two systems could not achieve complete synchronization under a large coupling strength [173], but synchronous motion with parallel offset could be realized, as shown in Figure 7. Based on the above dimensionality reduction reconstitution method, in the study of the synchronization of the memristor-coupled system, the inherent state initial mismatches between two identically coupled systems can be expressed as the initials-related parameter mismatches between two nonidentically coupled dimensionality reduction systems, and then, the quantitative theoretical research on the influence of the state initial on synchronization can be easily realized.

\section{Summary and Prospect}

The inherent memory property of the memristor makes the memristor-based circuit and system easy to produce the state initial-dependent dynamical behavior. Especially, the state initial-dependent extreme multistability phenomenon has been paid more and more attention by scholars, and abundant results have been obtained. Most of the existing literatures verify this special phenomenon through numerical simulation or circuit simulation, or capture different attractors randomly by closing and disconnecting the power supply in hardware experiments. The dimensionality reduction analysis method proposed in the literature theoretically realizes the precise prediction, quantitative analysis, and physical control of extreme multistability. For the ideal memristor-based circuit and system, the incremental fluxcharge analysis method and incremental integral transformation method can effectively realize dimensionality reduction modeling and extreme multistability reconstitution of memristive circuits and systems, and then, physical control and mechanism exposition of extreme multistability can be realized through quantitative analysis. It can be seen from the existing research contents and results that although great progress has been made in the study of the state initialdependent dynamical behavior of memristive circuits and systems, there are still many problems to be studied, mainly focusing on the following seven aspects: (a) prediction and control of the nonideal memristor-based circuit and system by state initial; (b) how to model the dimensionality reduction of the memristive circuit and system with high order or complex nonlinear terms; (c) study on the influence of state initial on the dynamical behavior of the memristorcoupled circuit and system and neural electrical networks; (d) for different types of complex memristive systems (such 
as time-delay memristive system and fractional-order memristive system), how to carry out equivalent transformation and dimensionality reduction modeling, so as to realize the reconstitution of its state initial-dependent dynamical behaviors; (e) the multistability of the original memristive circuit and system can be reconstructed from the dimensionality reduction model constructed by the incremental flux-charge analysis method and incremental integral transformation method, only when the state initial is set as the origin. However, the dimensionality reduction model is usually a nonlinear system, and its state initial will have a great influence on the system. Therefore, when the state initial is set to nonzero, how to predict and control the multistability of the original memristive circuit and system; (f) at present, the dimensionality reduction methods are used to study continuous memristive systems, so how to study the multistability of discrete memristive systems is an urgent scientific problem to be solved; (g) it is also a scientific problem to be solved whether the extreme multistability system can be built with real memristor devices and tested experimentally to make the research method more practical.

\section{Data Availability}

The data used to support the findings of this study are available from the corresponding author upon request.

\section{Conflicts of Interest}

The authors declare that they have no conflicts of interest.

\section{Acknowledgments}

This research issue was supported by the Natural Science Foundation of Henan Province under Grant no. 202300410351, Key Scientific Research of Colleges and Universities in Henan Province under Grant no. 21A120007, and Key Technologies R\&D Program of Henan Province under Grant no. 212102210084.

\section{References}

[1] L. O. Chua, "The fourth element," Proceedings of the IEEE, vol. 100, no. 6, pp. 1920-1927, 2012.

[2] J. Kengne, A. N. Negou, and D. Tchiotsop, "Antimonotonicity, chaos and multiple attractors in a novel autonomous memristor-based jerk circuit," Nonlinear Dynamics, vol. 88, no. 4, pp. 2589-2608, 2017.

[3] V. T. Pham, C. Volos, S. Jafari, X. Wang, and S. Vaidyanathan, "Hidden hyperchaotic attractor in a novel simple memristive neural network," Optoelectronics and Advanced Materials, Rapid Communications, vol. 8, no. 1112, pp. 1157-1163, 2014.

[4] L. Zhou, C. H. Wang, and L. L. Zhou, "Generating four-wing hyperchaotic attractor and two-wing, three-wing, and fourwing chaotic attractors in 4D memristive system," Internatinal Journal of Bifurcation and Chaos, vol. 27, no. 2, Article ID 1750027, 2017.

[5] Q. Li, H. Zeng, and J. Li, "Hyperchaos in a 4D memristive circuit with infinitely many stable equilibria," Nonlinear Dynamics, vol. 79, no. 4, pp. 2295-2308, 2015.
[6] Q. Tan, Y. Zeng, and Z. Li, "A simple inductor-free memristive circuit with three line equilibria," Nonlinear $D y$ namics, vol. 94, no. 3, pp. 1585-1602, 2018.

[7] G. Y. Wang, F. Yuan, G. R. Chen, and Y. Zhang, "Coexisting multiple attractors and riddled basins of a memristive system," Chaos, vol. 28, Article ID 013125, 2018.

[8] G. Y. Peng, F. H. Min, and E. R. Wang, "Circuit implementation, synchronization of multistability, and image encryption of a four-wing memristive chaotic system," Journal of Electrical and Computer Engineering, vol. 2018, Article ID 8649294, 13 pages, 2018.

[9] B. Hu, Z.-H. Guan, G. Chen, and F. L. Lewis, "Multistability of delayed hybrid impulsive neural networks with application to associative memories," IEEE Transactions on Neural Networks and Learning Systems, vol. 30, no. 5, pp. 1537-1551, 2019.

[10] X. Chen, S. Qian, F. Yu et al., "Pseudorandom number generator based on three kinds of four-wing memristive hyperchaotic system and its application in image encryption," Complexity, vol. 2020, Article ID 8274685, 17 pages, 2020.

[11] F. Yu, Z. Zhang, H. Shen et al., "Design and FPGA implementation of a pseudo-random number generator based on a Hopfield neural network under electromagnetic radiation," Frontiers in Physics, vol. 9, Article ID 690651, 2021.

[12] M. Chen, M. Sun, B. Bao, H. Wu, Q. Xu, and J. Wang, "Controlling extreme multistability of memristor emulatorbased dynamical circuit in flux-charge domain," Nonlinear Dynamics, vol. 91, no. 2, pp. 1395-1412, 2018.

[13] A. N. Pisarchik and U. Feudel, "Control of multistability," Physics Reports, vol. 540, no. 4, pp. 167-218, 2014.

[14] L. M. Pecora and T. L. Carroll, "Pseudoperiodic driving: eliminating multiple domains of attraction using chaos," Physical Review Letters, vol. 67, no. 8, pp. 945-948, 1991.

[15] T. L. Carroll and L. M. Pecora, "Using chaos to keep periodmultiplied systems in phase," Physical Review E, vol. 48, no. 4, pp. 2426-2436, 1993.

[16] B. K. Goswami, "Controlled destruction of chaos in the multistable regime," Physical Review E, vol. 76, no. 1, Article ID 016219, 2007.

[17] A. N. Pisarchik and R. Jaimes-Reategui, "Control of basins of attraction in a multistable fiber laser," Physics Letters A, vol. 374, no. 2, pp. 228-234, 2009.

[18] S. Gadaleta and G. Dangelmayr, "Learning to control a complex multistable system,” Physical Review E, vol. 63, no. 3, Article ID 036217, 2001.

[19] Y. Jiang, "Trajectory selection in multistable systems using periodic drivings," Physics Letters A, vol. 264, no. 1, pp. 22-29, 1999.

[20] M. Chen, B. C. Bao, T. Jiang et al., "Flux-charge analysis of initial state-dependent dynamical behaviors of a memristor emulator-based Chua's circuit," International Journal of Bifurcation and Chaos, vol. 28, no. 10, Article ID 1850120, 2018.

[21] M. Chen, Y. Feng, H. Bao et al., "State variable mapping method for studying initial-dependent dynamics in memristive hyper-jerk system with line equilibrium," Chaos, Solitons \& Fractals, vol. 115, pp. 313-324, 2018.

[22] B. C. Bao, Z. Liu, and H. Leung, "Is memristor a dynamic element?” Electronics Letters, vol. 49, no. 24, pp. 1523-1525, 2013.

[23] B. C. Bao, C. L. Wang, H. G. Wu, and X. H. Qiao, "Dimensionality reduction modeling and characteristic analysis 
of memristive circuit," Acta Physica Sinica, vol. 63, no. 2, Article ID 020504, 2014.

[24] B. C. Bao, F. W. Hu, Z. Liu, and J. P. Xu, "Mapping equivalent approach to analysis and realization of memristor based dynamical circuit," Chinese Physics B, vol. 23, no. 7, pp. 303-310, 2014.

[25] F. Yuan, G. Y. Wang, and X. W. Wang, "Chaotic oscillator containing memcapacitor and meminductor and its dimensionality reduction analysis," Chaos, vol. 27, no. 3, Article ID 033103, 2017.

[26] F. Corinto and M. Forti, "Memristor circuits: flux-charge analysis method," IEEE Transactions on Circuits and Systems I: Regular Papers, vol. 63, no. 11, pp. 1997-2009, 2016.

[27] F. Corinto and M. Forti, "Memristor circuits: bifurcations without parameters," IEEE Transactions on Circuits and Systems I: Regular Papers, vol. 64, no. 6, pp. 1540-1551, 2017.

[28] M. Di Marco, M. Forti, and L. Pancioni, "Memristor standard cellular neural networks computing in the flux-charge domain," Neural Networks, vol. 93, pp. 152-164, 2017.

[29] F. Corinto and M. Forti, "Complex dynamics in arrays of memristor oscillators via the flux-charge method," IEEE Transactions on Circuits and Systems I: Regular Papers, vol. 65, no. 3, pp. 1040-1050, 2017.

[30] H. Bao, T. Jiang, K. B. Chu, M. Chen, Q. Xu, and B. C. Bao, "Memristor-based canonical Chua's circuit: extreme multistability in voltage-current domain and its controllability in flux-charge domain," Complexity, vol. 2018, Article ID 5935637, 13 pages, 2018.

[31] M. Chen, M. X. Sun, H. Bao, Y. H. Hu, and B. C. Bao, "Fluxcharge analysis of two-memristor-based Chua's circuit: dimensionality decreasing model for detecting extreme multistability," IEEE Transactions on Industrial Electronics, vol. 67, no. 3, pp. 2197-2206, 2019.

[32] M. Chen, Y. Feng, H. Bao, B. C. Bao, H. G. Wu, and Q. Xu, "Hybrid state variable incremental integral for reconstructing extreme multistability in memristive jerk system with cubic nonlinearity," Complexity, vol. 2019, Article ID 8549472, , 2019.

[33] A. S. Pikovsky, M. Rosenblum, and J. Kurths, Synchronization: A Universal Concept in Nonlinear Sciences, Cambridge University Press, Cambridge, UK, 2003.

[34] H. G. Wu, S. Y. Chen, and B. C. Bao, "Impulsive synchronization and initial value effect for a memristor-based chaotic system," Acta Physica Sinica, vol. 64, no. 3, Article ID 030501, 2015.

[35] F. Wu, P. Zhou, A. Alsaedi, T. Hayat, and J. Ma, "Synchronization dependence on initial setting of chaotic systems without equilibria," Chaos, Solitons \& Fractals, vol. 110, pp. 124-132, 2018.

[36] F.-q. Wu, J. Ma, and G.-d. Ren, "Synchronization stability between initial-dependent oscillators with periodical and chaotic oscillation," Journal of Zhejiang University-Science A, vol. 19, no. 12, pp. 889-903, 2018.

[37] J. Zhang and X. Liao, "Effects of initial conditions on the synchronization of the coupled memristor neural circuits," Nonlinear Dynamics, vol. 95, no. 2, pp. 1269-1282, 2019.

[38] U. Feudel, "Complex dynamics in multistable systems," International Journal of Bifurcation and Chaos, vol. 18, no. 6, pp. 1607-1626, 2008.

[39] S. Jafari, A. Ahmadi, A. J. M. Khalaf, H. R. Abdolmohammadi, V. T. Pham, and F. E. Alsaadi, "A new hidden chaotic attractor with extreme multi-stability," AEÜ-International Journal of Electronics and Communications, vol. 89, pp. 131-135, 2018.
[40] F. Yu, L. Liu, H. Shen et al., "Multistability analysis, coexisting multiple attractors, and FPGA implementation of YuWang four-wing chaotic system," Complexity, vol. 2020, Article ID 7530976, 16 pages, 2020.

[41] F. Yu, H. Shen, L. Liu et al., "CCII and FPGA realization: a multistable modified fourth-order autonomous Chua's chaotic system with coexisting multiple attractors," Complexity, vol. 2020, Article ID 5212601, 17 pages, 2020.

[42] F. Atteneave, "Multistability in perception," Scientific American, vol. 225, no. 6, pp. 62-71, 1971.

[43] F. T. Arecchi and F. Lisi, "Hopping mechanism generating $1 \mathrm{f}$ noise in nonlinear systems," Physical Review Letters, vol. 49, no. 2, pp. 94-98, 1982.

[44] F. T. Arecchi, R. Meucci, G. Puccioni, and J. Tredicce, "Experimental evidence of subharmonic bifurcations, multistability, and turbulence in a Q-switched gas laser," Physical Review Letters, vol. 49, no. 17, pp. 1217-1220, 1982.

[45] Q. Hui, W. M. Haddad, and J. M. Bailey, "Multistability, bifurcations, and biological neural networks: a synaptic drive firing model for cerebral cortex transition in the induction of general anesthesia," Nonlinear Analysis: Hybrid Systems, vol. 5, no. 3, pp. 554-572, 2011.

[46] A. Geltrude, K. A. Naimee, S. Euzzor, R. Meucci, F. T. Arecchi, and B. K. Goswami, "Feedback control of bursting and multistability in chaotic systems," Communications in Nonlinear Science and Numerical Simulation, vol. 17, no. 7, pp. 3031-3039, 2012.

[47] J. C. Sprott, X. Wang, and G. R. Chen, "Coexistence of point, periodic and strange attractors," Internatinal Journal of Bifurcation and Chaos, vol. 23, no. 5, Article ID 1350093, 2013.

[48] P. R. Sharma, M. D. Shrimali, A. Prasad, and U. Feudel, "Controlling bistability by linear augmentation," Physics Letters A, vol. 377, no. 37, pp. 2329-2332, 2013.

[49] C. B. Li and J. C. Sprott, "Multistability in a butterfly flow," International Journal of Bifurcation and Chaos, vol. 23, no. 12, pp. 1294-1296, 2014.

[50] C. B. Li and J. C. Sprott, "Multistability in the Lorenz system: a broken butterfly," International Journal of Bifurcation and Chaos, vol. 24, no. 10, Article ID 1450131, 2014.

[51] C. B. Li, W. Hu, J. C. Sprott, and X. Wang, "Multistability in symmetric chaotic systems," The European Physical Journal Special Topics, vol. 224, no. 5, pp. 1493-1506, 2015.

[52] A. P. Kuznetsov, S. P. Kuznetsov, E. Mosekilde, and N. V. Stankevich, "Co-existing hidden attractors in a radiophysical oscillator system," Journal of Physics A: Mathematical and Theoretical, vol. 48, no. 12, Article ID 125101, 2015.

[53] P. R. Sharma, M. D. Shrimali, A. Prasad, N. V. Kuznetsov, and G. A. Leonov, "Control of multistability in hidden attractors," The European Physical Journal Special Topics, vol. 224, no. 8, pp. 1485-1491, 2015.

[54] B. C. Bao, Q. D. Li, N. Wang, and Q. Xu, "Multistability in Chua's circuit with two stable node-foci," Chaos, vol. 26, no. 4, Article ID 043111, 2016.

[55] B. C. Bao, A. H. Hu, H. Bao, Q. Xu, M. Chen, and H. G. Wu, "Three-dimensional memristive Hindmarsh-Rose neuron model with hidden coexisting asymmetric behaviors," Complexity, vol. 2018, Article ID 3872573, 11 pages, 2018.

[56] M. S. Patel, U. Patel, A. Sen et al., "Experimental observation of extreme multistability in an electronic system of two coupled Rössler oscillators," Physical Review E, vol. 89, no. 2, Article ID 022918, 2014 
[57] C. Hens, S. K. Dana, and U. Feudel, "Extreme multistability: attractor manipulation and robustness," Chaos, vol. 25, no. 5, Article ID 053112, 2015.

[58] Y. Liu and Z. You, "Multi-stability and almost periodic solutions of a class of recurrent neural networks," Chaos, Solitons \& Fractals, vol. 33, no. 2, pp. 554-563, 2007.

[59] C. N. Ngonghala, U. Feudel, and K. Showalter, "Extreme multistability in a chemical model system," Physical Review E, vol. 83, no. 5, Article ID 056206, 2011.

[60] C. R. Hens, R. Banerjee, U. Feudel, and S. K. Dana, "How to obtain extreme multistability in coupled dynamical systems," Physical Review E, vol. 85, no. 3, Article ID 035202, 2012.

[61] Z. T. Zhusubaliyev and E. Mosekilde, "Multistability and hidden attractors in a multilevel DC/DC converter," Mathematics and Computers in Simulation, vol. 109, pp. 32-45, 2015.

[62] M. Chen, J. Yu, and B. C. Bao, "Finding hidden attractors in improved memristor-based Chua's circuit," Electronics Letters, vol. 51, no. 6, pp. 462-464, 2015.

[63] G. A. Leonov, N. V. Kuznetsov, and V. I. Vagaitsev, "Localization of hidden Chua's attractors," Physics Letters A, vol. 375, no. 23, pp. 2230-2233, 2011.

[64] G. A. Leonov and N. V. Kuznetsov, "Hidden attractors in dynamical systems: from hidden oscillations in HilbertKolmogorov, Aizerman, and Kalman problems to hidden chaotic attractor in Chua circuits," International Journal of Bifurcation and Chaos, vol. 23, no. 1, Article ID 1330002, 2013.

[65] N. V. Kuznetsov and G. A. Leonov, "Hidden attractors in dynamical systems: systems with no equilibria, multistability and coexisting attractors," IFAC Proceedings Volumes, vol. 47, no. 3, pp. 5445-5454, 2014.

[66] Q. D. Li, H. Z. Zeng, and X. S. Yang, "On hidden twin attractors and bifurcation in the Chua's circuit," Nonlinear Dynamics, vol. 77, no. 1-2, pp. 255-266, 2014.

[67] B. C. Bao, F. W. Hu, M. Chen, Q. Xu, and Y. J. Yu, "Selfexcited and hidden attractors found simultaneously in a modified Chua's circuit," International Journal of Bifurcation and Chaos, vol. 25, no. 5, Article ID 1550075, 2015.

[68] V.-T. Pham, C. Volos, S. Jafari, and T. Kapitaniak, "Coexistence of hidden chaotic attractors in a novel no-equilibrium system," Nonlinear Dynamics, vol. 87, no. 3, pp. 2001-2010, 2017.

[69] S. Zhang and Y. Zeng, "A simple Jerk-like system without equilibrium: asymmetric coexisting hidden attractors, bursting oscillation and double full Feigenbaum remerging trees," Chaos, Solitons \& Fractals, vol. 120, pp. 25-40, 2019.

[70] M. Chen, C. Wang, H. Bao, X. Ren, and B. C. Bao, "Reconstitution for interpreting hidden dynamics with stable equilibrium point," Chaos, Solitons and Fractals, vol. 140, Article ID 110188, 2020.

[71] H. Chang, Y. X. Li, G. R. Chen, and F. Yuan, "Extreme multistability and complex dynamics of a memristor-based chaotic system," International Journal of Bifurcation and Chaos, vol. 30, no. 8, Article ID 2030019, 2020.

[72] Z. Wei, A. Yousefpour, H. Jahanshahi, U. Erkin Kocamaz, and I. Moroz, "Hopf bifurcation and synchronization of a five-dimensional self-exciting homopolar disc dynamo using a new fuzzy disturbance-observer-based terminal sliding mode control," Journal of the Franklin Institute, vol. 358, no. 1, pp. 814-833, 2021.

[73] N. Wang, G. S. Zhang, N. V. Kuznetsov, and H. Bao, "Hidden attractors and multistability in a modified Chua's circuit,"
Communication in Nonlinear Science and Numerical Simulation, vol. 92, Article ID 105494, 2021.

[74] C. Du, L. Liu, Z. Zhang, and S. Yu, "A Coupling method of double memristors and analysis of extreme transient behavior," Nonlinear Dynamics, vol. 104, no. 1, pp. 765-787, 2021.

[75] X. L. An and S. Qiao, “The hidden, period-adding, mixedmode oscillations and control in a HR neoron under electromagnetic induction," Chaos, Solitons and Fractals, vol. 143, Article ID 110587, 2021.

[76] H. Jahanshahi, O. O. Lopez, J. M. M. Pacheco et al., "Simulation and experimental validation of a non-equilibrium chaotic system," Chaos, Solitons and Fractals, vol. 143, Article ID 110539, 2021.

[77] T. M. Liu, H. Z. Yan, S. Banerjee, and J. Mou, “A Fractionalorder chaotic system with hidden attractor and self-excited attractor and its DSP implementation," Chaos, Solitons and Fractals, vol. 145, Article ID 110791, 2021.

[78] D. Dudkowski, S. Jafari, T. Kapitaniak, N. V. Kuznetsov, G. A. Leonov, and A. Prasad, "Hidden attractors in dynamical systems," Physics Reports, vol. 637, pp. 1-50, 2016.

[79] S. Zhang, J. H. Zheng, X. P. Wang, and Z. G. Zeng, “A novel no-equilibrium HR neuron model with hidden homogeneous extreme multistability," Chaos, Solitons and Fractals, vol. 145, Article ID 110761, 2021.

[80] Y. Yang, L. L. Huang, J. H. Xiang, H. Bao, and H. Z. Li, "Design of multi-wing 3D chaotic systems with only stable equilibrium or no equilibrium point using rotation symmetry," AEÜ-International Journal of Electronics and Communications, vol. 135, Article ID 153710, 2021.

[81] V. T. Pham, S. Jafari, S. Vaidyanathan, C. Volos, and $\mathrm{X}$. Wang, "A novel memristive neural network with hidden attractors and its circuitry implementation," Science China Technological Sciences, vol. 59, no. 3, pp. 358-363, 2016.

[82] L. O. Chua and S. M. Sung Mo Kang, "Memristive devices and systems," Proceedings of the IEEE, vol. 64, no. 2, pp. 209-223, 1976.

[83] B. C. Bao, J. P. Xu, and Z. Liu, "Initial state dependent dynamical behaviors in memristor based chaotic circuit," Chinese Physics Letters, vol. 27, no. 7, Article ID 070504, 2010.

[84] B. C. Bao, Z. H. Ma, J. P. Xu, Z. Liu, and Q. Xu, "A simple memristor chaotic circuit with complex dynamics," International Journal of Bifurcation and Chaos, vol. 21, no. 9, pp. 2629-2645, 2011.

[85] B. C. Bao, J. P. Xu, G. H. Zhou, Z. H. Ma, and L. Zou, "Chaotic memristive circuit: equivalent circuit realization and dynamical analysis," Chinese Physics B, vol. 20, no. 12, Article ID 120502, 2011.

[86] B. Bao, G. Shi, J. Xu, Z. Liu, and S. Pan, "Dynamics analysis of chaotic circuit with two memristors," Science China Technological Sciences, vol. 54, no. 8, pp. 2180-2187, 2011.

[87] B. C. Bao, X. Zhou, Z. Liu, and F. W. Hu, "Generalized memory element and chaotic memory system," International Journal of Bifurcation and Chaos, vol. 23, no. 8, Article ID 1350135, 2013.

[88] B. C. Bao, Q. Xu, H. Bao, and M. Chen, "Extreme multistability in a memristive circuit," Electronics Letters, vol. 52, no. 12, pp. 1008-1010, 2016.

[89] S. Jafari, A. Ahmadi, S. Panahi, and K. Rajagopal, "Extreme multi-stability: when imperfection changes quality," Chaos, Solitons \& Fractals, vol. 108, pp. 182-186, 2018. 
[90] D. B. Strukov, G. S. Snider, D. R. Stewart, and R. S. Williams, "The missing memristor found," Nature, vol. 453, no. 7191, pp. 80-83, 2008.

[91] S. Duan, X. Hu, Z. Dong, L. Wang, and P. Mazumder, "Memristor-based cellular nonlinear/neural network: design, analysis, and applications," IEEE Transactions on Neural Networks and Learning Systems, vol. 26, no. 6, pp. 1202-1213, 2015.

[92] Y. Babacan, F. Kaçar, and K. Gürkan, "A spiking and bursting neuron circuit based on memristor," Neurocomputing, vol. 203, pp. 86-91, 2016.

[93] B. Bao, T. Jiang, Q. Xu, M. Chen, H. Wu, and Y. Hu, "Coexisting infinitely many attractors in active band-pass filter-based memristive circuit," Nonlinear Dynamics, vol. 86, no. 3, pp. 1711-1723, 2016.

[94] J. Zhang and X. Liao, "Synchronization and chaos in coupled memristor-based Fitz Hugh-Nagumo circuits with memristor synapse," AEU-International Journal of Electronics and Communications, vol. 75, pp. 82-90, 2017.

[95] G. M. Tornez Xavier, F. Gómez Castañeda, L. M. Flores Nava, and J. A. Moreno Cadenas, "Memristive recurrent neural network," Neurocomputing, vol. 273, pp. 281-295, 2018.

[96] H. Bao, N. Wang, B. Bao, M. Chen, P. Jin, and G. Wang, "Initial condition-dependent dynamics and transient period in memristor-based hypogenetic jerk system with four line equilibria," Communications in Nonlinear Science and $\mathrm{Nu}$ merical Simulation, vol. 57, pp. 264-275, 2018.

[97] C. Qin, K. H. Sun, and S. B. He, "Characterstic analysis of fractional-order memristor-based hypogenetic jerk system and its DSP implementation," Electronics, vol. 10, no. 7, Article ID 841, 2021.

[98] B. A. Mezatio, M. T. Motchongom, B. R. Wafo Tekam, R. Kengne, R. Tchitnga, and A. Fomethe, "A novel memristive 6D hyperchaotic autonomous system with hidden extreme multistability," Chaos, Solitons \& Fractals, vol. 120, pp. 100-115, 2019.

[99] W. Wang, Y. C. Zeng, and R. T. Sun, "Research on a six-order chaotic circuit with three memristors," Acta Physica Sinica, vol. 66, no. 4, Article ID 040502, 2017.

[100] B. C. Bao, M. A. Peol, H. Bao, M. Chen, H. Z. Li, and B. Chen, "No-argument memristive hyper-jerk system and its coexisting chaotic bubbles boosted by initial conditions," Chaos, Solitons and Fractals, vol. 144, Article ID 110744, 2021.

[101] Q. Xu, Y. Lin, B. Bao, and M. Chen, "Multiple attractors in a non-ideal active voltage-controlled memristor based Chua's circuit," Chaos, Solitons \& Fractals, vol. 83, pp. 186-200, 2016.

[102] M. Chen, Q. Xu, Y. Lin, and B. Bao, "Multistability induced by two symmetric stable node-foci in modified canonical Chua's circuit," Nonlinear Dynamics, vol. 87, no. 2, pp. 789-802, 2017.

[103] B. C. Bao, H. Bao, N. Wang, M. Chen, and Q. Xu, "Hidden extreme multistability in memristive hyperchaotic system," Chaos, Solitons \& Fractals, vol. 94, pp. 102-111, 2017.

[104] Z. Wang, A. Akgul, V.-T. Pham, and S. Jafari, "Chaos-based application of a novel no-equilibrium chaotic system with coexisting attractors," Nonlinear Dynamics, vol. 89, no. 3, pp. 1877-1887, 2017.

[105] J. Kengne, Z. N. Tabekoueng, V. K. Tamba, and A. N. Negou, "Periodicity, chaos, and multiple attractors in a memristorbased Shinriki's circuit," Chaos, vol. 25, no. 10, Article ID 103126, 2015.
[106] G. Y. Wang, C. B. Shi, X. W. Wang, and F. Yuan, "Coexisting oscillation and extreme multistability for a memcapacitorbased circuit," Mathematical Problems in Engineering, vol. 2017, Article ID 6504969, 13 pages, 2017.

[107] F. Yuan, G. Y. Wang, and X. W. Wang, "Extreme multistability in a memristor-based Chua's multi-scroll hyperchaotic system," Chaos, vol. 26, no. 7, Article ID 073107, 2016.

[108] Y. M. Lu and F. H. Min, "Dynamic analysis of symmetric behavior in flux-controlled memristor circuit based on field programmable gate array," Acta Physica Sinica, vol. 68, no. 13, Article ID 130502, 2019.

[109] H. M. Li, Y. F. Yang, W. Li, S. B. He, and C. L. Li, "Extremely rich dynamics in a memristor-based chaotic system," European Physical Journal Plus, vol. 135, no. 7, Article ID 579, 2020.

[110] Y. Zhang, Z. Liu, H. Wu, S. Chen, and B. Bao, "Twomemristor-based chaotic system and its extreme multistability reconstitution via dimensionality reduction analysis," Chaos, Solitons \& Fractals, vol. 127, pp. 354-363, 2019.

[111] C. Li, J. C. Sprott, and Y. Mei, "An infinite 2-D lattice of strange attractors," Nonlinear Dynamics, vol. 89, no. 4, pp. 2629-2639, 2017.

[112] C. Li, W. Joo-Chen Thio, J. C. Sprott, H. H.-C. Iu, and Y. Xu, "Constructing infinitely many attractors in a programmable chaotic circuit," IEEE Access, vol. 6, pp. 29003-29012, 2018.

[113] C. Li and J. C. Sprott, "An infinite 3-D quasiperiodic lattice of chaotic attractors," Physics Letters A, vol. 382, no. 8, pp. 581-587, 2018.

[114] H. G. Wu, Y. Ye, B. C. Bao, M. Chen, and Q. Xu, "Memristor initial boosting behaviors in a two-memristor-based hyperchaotic system," Chaos, Solitons \& Fractals, vol. 121, pp. 178-185, 2019.

[115] L. Chua, "Memristor-the missing circuit element," IEEE Transactions on Circuit Theory, vol. 18, no. 5, pp. 507-519, 1971.

[116] L. Chua, "Resistance switching memories are memristors," Applied Physics A, vol. 102, no. 4, pp. 765-783, 2011.

[117] B. C. Bao, Q. Xu, and H. Bao, Memristive Circuit and Multistability, Science Press, Beijing, China, 2018.

[118] B. Muthuswamy, "Implementing memristor based chaotic circuits," International Journal of Bifurcation and Chaos, vol. 20, no. 5, pp. 1335-1350, 2010.

[119] H. H. C. Iu, D. S. Yu, A. L. Fitch, V. Sreeram, and H. Chen, "Controlling chaos in a memristor based circuit using a twinT notch filter," IEEE Transactions on Circuits and Systems I: Regular Papers, vol. 58, no. 6, pp. 1337-1344, 2011.

[120] H. G. Wu, B. C. Bao, Z. Liu, Q. Xu, and P. Jiang, "Chaotic and periodic bursting phenomena in a memristive Wien-bridge oscillator," Nonlinear Dynamics, vol. 83, no. 1-2, pp. 893-903, 2016.

[121] B. C. Bao, P. Wu, H. Bao, M. Chen, and Q. Xu, "Chaotic bursting in memristive diode bridge-coupled Sallen-key lowpass filter," Electronics Letters, vol. 53, no. 16, pp. 1104-1105, 2017.

[122] Q. Quan Xu, N. Wang, B. C. Ning Wang, M. Bocheng Bao, C. D. Mo Chen, and C. Li, "A feasible memristive Chua's circuit via bridging a generalized memristor," Journal of Applied Analysis \& Computation, vol. 6, no. 4, pp. 1152-1163, 2016.

[123] M. Chen, J. Yu, Q. Yu, C. Li, and B. Bao, "A memristive diode bridge-based canonical Chua's circuit," Entropy, vol. 16, no. 12, pp. 6464-6476, 2014. 
[124] B. C. Bao, P. Y. Wu, H. Bao, Q. Xu, and M. Chen, "Numerical and experimental confirmations of quasi-periodic behavior and chaotic bursting in third-order autonomous memristive oscillator," Chaos, Solitons \& Fractals, vol. 106, pp. 161-170, 2018.

[125] L. O. Chua, "Nonlinear circuit foundations for nanodevices, part I: the four-element torus," Proceedings of the IEEE, vol. 9, no. 11, pp. 1830-1859, 2003.

[126] R. Williams, "How we found the missing memristor," IEEE Spectrum, vol. 45, no. 12, pp. 28-35, 2008.

[127] D. Biolek, V. Biolkova, and Z. Biolek, "Pinched hysteretic loops of ideal memristors, memcapacitors and meminductors must be "self-crossing"” Electronics Letters, vol. 47, no. 25, pp. 1385-1387, 2011.

[128] S. P. Adhikari, M. P. Sah, H. Kim, and L. O. Chua, “Three fingerprints of memristor," IEEE Transactions on Circuits and Systems I: Regular Papers, vol. 60, no. 11, pp. 3008-3021, 2013.

[129] Y. Lin, W. B. Liu, and Q. Shen, "Bi-stability in a fifth-order voltage-controlled memristor-based Chua's chaotic circuit," Acta Physica Sinica, vol. 67, no. 23, Article ID 230502, 2018.

[130] B. Bao, T. Jiang, G. Wang, P. Jin, H. Bao, and M. Chen, “Twomemristor-based Chua's hyperchaotic circuit with plane equilibrium and its extreme multistability," Nonlinear Dynamics, vol. 89, no. 2, pp. 1157-1171, 2017.

[131] L. Wang, S. Zhang, Y. C. Zeng, and Z. J. Li, “Generating hidden extreme multistability in memristive chaotic oscillator via micro-perturbation," Electronics Letters, vol. 54, no. 13, pp. 808-810, 2018.

[132] T. Fozin Fonzin, J. Kengne, and F. B. Pelap, "Dynamical analysis and multistability in autonomous hyperchaotic oscillator with experimental verification," Nonlinear Dynamics, vol. 93, no. 2, pp. 653-669, 2018.

[133] S. Morfu, B. Nofiele, and P. Marquié, "On the use of multistability for image processing," Physics Letters A, vol. 367, no. 3, pp. 192-198, 2007.

[134] G. Peng and F. Min, "Multistability analysis, circuit implementations and application in image encryption of a novel memristive chaotic circuit," Nonlinear Dynamics, vol. 90, no. 3, pp. 1607-1625, 2017.

[135] J. Sun, Q. Yang, and Y. Wang, "Dynamical analysis of novel memristor chaotic system and DNA encryption application," Iranian Journal of Science and Technology, Transactions of Electrical Engineering, vol. 44, no. 1, pp. 449-460, 2020.

[136] S. Zhang, J. H. Zheng, X. P. Wang, and Z. G. Zeng, "Multiscroll hidden attractor in memristive HR neuron model under electromagnetic radiation and its applications," Chaos, vol. 31, no. 1, Article ID 011101, 2021.

[137] Q. Lai, Z. Wan, A. Akgul, O. F. Boyraz, and M. Z. Yildiz, "Design and implementation of a new memristive chaotic system with application in touchless fingerprint encryption," Chinese Journal of Physics, vol. 67, pp. 615-630, 2020.

[138] F. Z. Wang, "A triangular periodic table of elementary circuit elements," IEEE Transactions on Circuits and Systems I: Regular Papers, vol. 60, no. 3, pp. 616-623, 2013.

[139] J. Mou, K. Sun, J. Ruan, and S. He, "A nonlinear circuit with two memcapacitors," Nonlinear Dynamics, vol. 86, no. 3, pp. 1735-1744, 2016.

[140] Y. Z. Zhang, Z. Liu, H. G. Wu, S. Y. Chen, and B. C. Bao, "Extreme multistability in memristive hyper-jerk system and stability mechanism analysis using dimensionality reduction model," European Physical Journal Special Topic, vol. 228, no. 10, pp. 1995-2009, 2019.
[141] H. Bao, W. Liu, and M. Chen, "Hidden extreme multistability and dimensionality reduction analysis for an improved non-autonomous memristive FitzHugh-Nagumo circuit," Nonlinear Dynamics, vol. 96, no. 3, pp. 1879-1894, 2019.

[142] C. K. Volos, I. M. Kyprianidis, I. M. Kyprianidis, I. N. Stouboulos, E. Tlelo-Cuautle, and S. Vaidyanathan, "Memristor: a new concept in synchronization of coupled neuromorphic circuits," Journal of Engineering Science and Technology Review, vol. 8, no. 2, pp. 157-173, 2015.

[143] Y. Xu, Y. Jia, J. Ma, T. Hayat, and A. Alsaedi, "Collective response in electrical activities of neurons under field coupling," Scientific Reports, vol. 8, no. 1, Article ID 1349, 2018.

[144] M. Ge, Y. Jia, Y. Xu, and L. Yang, "Mode transition in electrical activities of neuron driven by high and low frequency stimulus in the presence of electromagnetic induction and radiation," Nonlinear Dynamics, vol. 91, no. 1, pp. 515-523, 2018.

[145] Z. Wang, S. Joshi, S. E. Savel'ev et al., "Memristors with diffusive dynamics as synaptic emulators for neuromorphic computing," Nature Materials, vol. 16, no. 1, pp. 101-108, 2017.

[146] H. Kim, M. P. Sah, C. Yang, T. Roska, and L. O. Chua, "Memristor bridge synapses," Proceedings of the IEEE, vol. 100, no. 6, pp. 2061-2070, 2012.

[147] X. S. Yang, J. D. Cao, and J. L. Liang, "Exponential synchronization of memristive neural networks with delays: interval matrix method," IEEE Transactions on Neural Networks and Learning Systems, vol. 28, no. 8, pp. 1878-1888, 2016.

[148] C. H. Wang, H. R. Lin, J. R. Sun, L. Zhou, C. Zhou, and Q. L. Deng, "Research progress on chaos, memory and neural network circuits based on memristor," Journal of Electronics and Information Technology, vol. 42, no. 4, pp. 795-810, 2020.

[149] D. Ma, G. Wang, C. Han, Y. Shen, and Y. Liang, “A memristive neural network model with associative memory for modeling affections," IEEE Access, vol. 6, pp. 6161461622,2018

[150] H. Bao, W. Liu, and A. Hu, "Coexisting multiple firing patterns in two adjacent neurons coupled by memristive electromagnetic induction," Nonlinear Dynamics, vol. 95, no. 1 , pp. 43-56, 2019

[151] C. Chen, J. Chen, H. Bao, M. Chen, and B. Bao, "Coexisting multi-stable patterns in memristor synapse-coupled Hopfield neural network with two neurons," Nonlinear Dynamics, vol. 95, no. 4, pp. 3385-3399, 2019.

[152] M. Chen, C. J. Chen, B. C. Bao, and Q. Xu, "Initial sensitive dynamics in memristor synapse-coupled Hopfield neural network," Journal of Electronics and Information Technology, vol. 42, no. 4, pp. 870-877, 2020.

[153] S. Mostaghimi, F. Nazarimehr, S. Jafari, and J. Ma, "Chemical and electrical synapse-modulated dynamical properties of coupled neurons under magnetic flow," Applied Mathematics and Computation, vol. 348, pp. 42-56, 2019.

[154] K. Wu, T. Wang, C. Wang, T. Du, and H. Lu, "Study on electrical synapse coupling synchronization of HindmarshRose neurons under Gaussian white noise," Neural Computing and Applications, vol. 30, no. 2, pp. 551-561, 2018.

[155] M. Ge, Y. Jia, J. B. Kirunda et al., "Propagation of firing rate by synchronization in a feed-forward multilayer HindmarshRose neural network," Neurocomputing, vol. 320, pp. 60-68, 2018. 
[156] G. Ren, Y. Xu, and C. Wang, "Synchronization behavior of coupled neuron circuits composed of memristors," Nonlinear Dynamics, vol. 88, no. 2, pp. 893-901, 2017.

[157] W. Yao, C. Wang, J. Cao, Y. Sun, and C. Zhou, "Hybrid multisynchronization of coupled multistable memristive neural networks with time delays," Neurocomputing, vol. 363, pp. 281-294, 2019.

[158] R. Eckhorn, "Neural mechanisms of scene segmentation: recordings from the visual cortex suggest basic circuits for linking field models," IEEE Transactions on Neural Networks, vol. 10, no. 3, pp. 464-479, 1999.

[159] R. Bartsch, J. W. Kantelhardt, T. Penzel, and S. Havlin, "Experimental evidence for phase synchronization transitions in the human cardiorespiratory system," Physical Review Letters, vol. 98, Article ID 54102, 2007.

[160] P. J. Uhlhaas and W. Singer, "Neural synchrony in brain disorders: relevance for cognitive dysfunctions and pathophysiology," Neuron, vol. 52, no. 1, pp. 155-168, 2006.

[161] F. Yu, L. Liu, B. Y. He et al., "Analysis and FPGA realization of a novel 5D hyperchaotic four-wing memristive system, active control synchronization, and secure communication application," Complexity, vol. 2019, Article ID 4047957, 18 pages, 2019.

[162] F. Yu, L. Liu, H. Shen et al., "Dynamic analysis, circuit design and synchronization of a novel $6 \mathrm{D}$ memristive four-wing hyperchaotic system with multiple coexisting attractors," Complexity, vol. 2020, Article ID 5904607, 17 pages, 2020.

[163] S. Boccaletti, J. Kurths, G. Osipov, D. L. Valladares, and C. S. Zhou, "The synchronization of chaotic systems," Physical Reports, vol. 366, no. 1, pp. 1-101, 2002.

[164] Q. Y. Wang, Z. S. Duan, Z. S. Feng, G. R. Chen, and Q. S. Lu, "Synchronization transition in gap-junction-coupled leech neurons," Physica A, vol. 387, no. 16-17, pp. 4404-4410, 2008.

[165] Y. Xu, Y. Jia, J. Ma, A. Alsaedi, and B. Ahmad, "Synchronization between neurons coupled by memristor," Chaos, Solitons \& Fractals, vol. 104, pp. 435-442, 2017.

[166] F. Xu, J. Zhang, T. Fang, S. Huang, and M. Wang, "Synchronous dynamics in neural system coupled with memristive synapse," Nonlinear Dynamics, vol. 92, no. 3, pp. 1395-1402, 2018.

[167] X. S. Yang and D. W. C. Ho, "Synchronization of delayed memristive neural networks: robust analysis approach," IEEE Transactions on Cybernetics, vol. 46, no. 12, pp. 3377-3387, 2015.

[168] X. Xiong, R. Tang, and X. Yang, "Finite-time synchronization of memristive neural networks with proportional delay," Neural Processing Letters, vol. 50, no. 2, pp. 1139-1152, 2018.

[169] H. Bao, Y. Zhang, W. Liu, and B. Bao, "Memristor synapsecoupled memristive neuron network: synchronization transition and occurrence of chimera," Nonlinear Dynamics, vol. 100, no. 1, pp. 937-950, 2020.

[170] B. Bao, Q. Yang, D. Zhu, Y. Zhang, Q. Xu, and M. Chen, "Initial-induced coexisting and synchronous firing activities in memristor synapse-coupled Morris-Lecar bi-neuron network," Nonlinear Dynamics, vol. 99, no. 3, pp. 2339-2354, 2020.

[171] Q. Lai, Z. Q. Wan, P. D. K. Kuate, and H. Fotsin, "Coexisting attractors, circuit implementation and synchronization control of a new chaotic system evolved from the simplest memristor chaotic circuit," Communications in Nonlinear Science and Numerical Simulation, vol. 89, Article ID 105341, 2020.
[172] F. Min, C. Li, L. Zhang, and C. Li, "Initial value-related dynamical analysis of the memristor-based system with reduced dimensions and its chaotic synchronization via adaptive sliding mode control method," Chinese Journal of Physics, vol. 58, pp. 117-131, 2019.

[173] Y. Zhang, Z. Liu, H. Wu, S. Chen, and B. Bao, "Dimensionality reduction analysis for detecting initial effects on synchronization of memristor-coupled system," IEEE Access, vol. 7, no. 1, pp. 109689-109698, 2019. 